DESY 04-064

hep-ph/0701019

SFB/CPP-06-63

December 2006

\title{
Universal Higher Order Singlet QED \\ Corrections to Unpolarized Lepton Scattering
}

\author{
Johannes Blümlein $^{a}$ and Hiroyuki Kawamura ${ }^{a, b}$ \\ ${ }^{a}$ Deutsches Elektronen-Synchrotron, DESY, \\ Platanenallee 6, D-15738 Zeuthen, Germany \\ ${ }^{b}$ Radiation Laboratory, RIKEN, Wako 351-0198, Japan
}

\begin{abstract}
We calculate the universal flavor-singlet radiative QED corrections to unpolarized lepton scattering applicable to general differential scattering cross sections, involving charged fermions or photons in initial or final states. The radiators are derived to $O\left(\left(\alpha \ln \left(Q^{2} / m_{f}^{2}\right)\right)^{5}\right)$ in analytic form. Numerical illustrations are given.
\end{abstract}




\section{Introduction}

QED corrections to integral and differential cross sections of light charged lepton-anti-lepton scattering or deeply inelastic lepton-nucleon scattering turn out to be quite large in some kinematic regions [1-4]. This applies in particular to the Bremsstrahlung contributions due to significant shifts in the kinematics of the underlying differential scattering cross sections. The universal corrections can be grouped into flavor non-singlet and flavor singlet contributions. In the orders $O\left(\left(e_{f}^{2} \alpha L\right)^{k}\right)$, with $\alpha$ the fine structure constant, $L=\ln \left(Q^{2} / m_{f}^{2}\right), Q^{2}$ the typical virtuality of the process and $m_{f}, e_{f}$ the fermion mass and charge, respectively, the non-singlet contributions stem from the leading order anomalous dimension in QED, $P_{f f}\left(x, Q^{2}\right)$. The diagonal elements of the singlet anomalous dimension matrix contain a $\delta(1-x)$-distribution and are distribution-valued due to $(\ldots)_{+}$-distributions. Therefore the numerical Mellin-inversion does badly converge in the region of $x \lesssim 1$. Analytic representations are required to high enough order $k$ in the fine structure constant $\alpha$ to cover all universal effects for the energy ranges probed at present day colliders and those to be built in the foreseeable future. This applies to high luminosity experiments at future linear colliders [5] and as well to the search for rare reactions at LHC. The second order universal corrections for various processes are known for a long time [6-9]. The 3rd order corrections were given in [10] ${ }^{1}$ for the flavor non-singlet and in [12] also for the singlet case. Later the 5 th order non-singlet corrections were given in [13] and recalculated in $[14]$ and $[15,16]^{2}$. In Ref. [15] a very compact form was given for the non-singlet contributions, which are the same for polarized and unpolarized scattering. There also the polarized singlet contributions were calculated to $O\left((\alpha L)^{5}\right)$. In the present paper the unpolarized singlet evolution kernels are calculated to $O\left((\alpha L)^{5}\right)$ which supplements earlier investigations for the flavor non-singlet kernel [15]. A second class of universal QED corrections was treated previously in the non-singlet [17] and polarized singlet case [15]. It concerns the leading order small $x$ resummations of $O\left(\left(\alpha \ln ^{2}(x)\right)^{k}\right)$. These resummations are based on corresponding resummations in QCD [18]. In the unpolarized (pure) singlet case the leading order small- $x$ QCD resummations [19] result from the non-abelian gluon coupling, which is absent in QED. Therefore a transformation of the respective kernels is not possible in this case.

The paper is organized as follows. The general framework to derive the radiators in $O\left((\alpha L)^{k}\right)$ is outlined in section 2. In section 3 the leading order singlet radiators are calculated to $O\left((\alpha L)^{5}\right)$. In section 4 numerical illustrations are given and section 5 contains the conclusions. An appendix lists useful convolution relations which were needed in the calculation and are of use in other QED and QCD calculations.

\section{The Solution of Singlet Evolution Equations}

The universal QED corrections $O\left((\alpha L)^{k}\right)$ for general values of the collinear radiation momentum fraction $x$ can be expressed solving the singlet evolution equations starting at a low scale $Q_{0}^{2}$. This scale may be identified with a typical charged lepton mass $m_{f}$ squared. The running coupling constant $a\left(Q^{2}\right)=\alpha\left(Q^{2}\right) /(4 \pi)$ obeys the evolution equation

$$
\frac{d a\left(Q^{2}\right)}{d \ln \left(Q^{2}\right)}=-\sum_{k=0}^{\infty} \beta_{k} a^{k+2}\left(Q^{2}\right),
$$

\footnotetext{
${ }^{1}$ For an application to the $Z$-peak see [11].

${ }^{2}$ The results of [13] and [15] agree but partly disagree in the 5th order with [14].
} 
where $\beta_{k}$ denote the expansion coefficients of the QED $\beta$-function in the $\overline{\mathrm{MS}}$-scheme, $\beta_{0}=$ $-4 / 3 N_{f}, \beta_{1}=-4 N_{f}, \beta_{2}=2 N_{f}+(44 / 9) N_{f}^{2}$ etc. [20] for $N_{f}$ active charged lepton species. At leading order the solution

$$
a\left(Q^{2}\right)=\frac{a\left(m_{f}^{2}\right)}{1-\frac{4}{3} N_{f} a\left(m_{f}^{2}\right) L},
$$

with $L=\ln \left(Q^{2} / m_{f}^{2}\right)$ is obtained.

The universal radiators are found as solutions of the leading order QED renormalization group equations associated to the collinear singularities. The following QED-distributions are of relevance :

$$
\begin{aligned}
D_{\mathrm{NS}}^{f}(a, x) & =D^{f}(a, x)-D^{\bar{f}}(a, x) \\
D_{\Sigma}^{f}(a, x) & =D^{f}(a, x)+D^{\bar{f}}(a, x) \\
D_{\gamma \gamma}(a, x) & =D_{22}(a, x) \\
D_{\gamma f}(a, x) & =D_{21}(a, x) \\
D_{f \gamma}(a, x) & =D_{12}(a, x)
\end{aligned}
$$

The non-singlet distribution $D_{\mathrm{NS}}^{f}(a, x)$ was dealt with in [13-15] before. Here the $D_{i j}(a, x)$ denote the respective matrix element of the singlet radiator $\boldsymbol{D}_{S}(a, x)$ given below.

The singlet radiator functions at the scale $Q_{0}^{2}$ are

$$
\boldsymbol{D}\left(Q_{0}^{2}\right)(x) \equiv \boldsymbol{D}\left(a_{0}\right)=\mathbf{1} \quad \delta(1-x)
$$

since both the charged leptons and the photon are considered to be asymptotically stable particles. The evolution equations read

$$
\begin{aligned}
\frac{\partial \boldsymbol{D}_{S}(a, x)}{\partial a} & =-\frac{1}{a} \frac{\sum_{k=0}^{\infty} a^{k} \boldsymbol{P}_{k}(x)}{\sum_{k=0}^{\infty} a^{k} \beta_{k}} \otimes \boldsymbol{D}_{S}(a, x) \\
& =-\frac{1}{\beta_{0} a}\left[\boldsymbol{P}_{0}(x)+a\left(\boldsymbol{P}_{1}(x)-\frac{\beta_{1}}{\beta_{0}} \boldsymbol{P}_{0}(x)\right)+O\left(a^{2}\right)\right] \otimes \boldsymbol{D}_{S}(a, x) .
\end{aligned}
$$

The Mellin convolution is defined by

$$
A(x) \otimes B(x)=\int_{0}^{1} d x_{1} \int_{0}^{1} d x_{2} \delta\left(x-x_{1} x_{2}\right) A\left(x_{1}\right) B\left(x_{2}\right) .
$$

The Mellin transform

$$
\boldsymbol{M}[f(x)](N)=\int_{0}^{1} d x x^{N-1} f(x)
$$

diagonalizes the convolution (10) to

$$
\boldsymbol{M}[A(x) \otimes B(x)](N)=\boldsymbol{M}[A(x)](N) \cdot \boldsymbol{M}[B(x)](N) .
$$

In some of the radiators $(\ldots)_{+}$distributions emerge, which are defined relative to the set of smooth test functions $\phi(x)$ with compact support by

$$
\int_{0}^{1} d x[F(x)]_{+} \phi(x)=\int_{0}^{1} d x F(x)[\phi(x)-\phi(1)] .
$$


Eq. (9) may be solved easiest in Mellin space as a matrix-valued ordinary differential equation to all orders, see e.g. [21,22]. The leading order solution reads

$$
\boldsymbol{D}_{S, 0}(a, x)=\left[\exp \left(-\boldsymbol{R}_{0}(x) \ln \left(a / a_{0}\right)\right)_{\otimes}\right] \otimes \boldsymbol{D}_{S}\left(a_{0}, x\right) \equiv \boldsymbol{E}_{0}\left(a, a_{0}, x\right) \otimes \boldsymbol{D}_{S}\left(a_{0}, x\right),
$$

where $\boldsymbol{R}_{0}=\boldsymbol{P}_{0} / \beta_{0}$ and $\boldsymbol{E}_{0}$ denotes the leading order singlet evolution operator. We use the short-hand notation

$$
\left[f(g(x))_{\otimes}\right]=\sum_{k=0}^{\infty} \frac{f^{(k)}(0)}{k !} \otimes_{l=1}^{k} g(x),
$$

with $\otimes_{l=1}^{k}$ the $k$-fold convolution. The singlet solution (14) to $k$ th order in $\alpha\left(Q^{2}\right) L$ therefore requires to calculate $k$-fold convolutions of the leading order matrix of splitting functions.

The method described above can be extended to sub-leading logarithmic contributions, i.e. terms of $O\left(\alpha^{2} L\right)$ etc. These contributions contain process-dependent parts being described by Wilson coefficients in inclusive situations or the corresponding semi-inclusive quantities. These corrections are neither universal nor independent of the measurement chosen for the kinematic variables. Examples are the $O\left(\alpha^{2} L\right)$ corrections for the initial state radiation in $e^{+} e^{-}$annihilation [6], the $O\left(\alpha^{2} L\right)$ initial and final state radiation corrections to deeply inelastic scattering [23] and the the $O\left(\alpha^{2} \ln \left(m_{\mu} / m_{e}\right)\right)$ corrections to the electron spectrum in muon decay [24].

\section{The Leading Order Solution to $O\left[(\alpha L)^{5}\right]$}

In the following we derive the solution for the unpolarized singlet QED evolution kernels up to $O\left((\alpha L)^{5}\right)$. The singlet evolution equation is solved in the running coupling $a\left(Q^{2}\right)$. However, one may re-parameterize the representation and express the evolution kernel directly in terms of $a_{0}=a\left(m_{f}^{2}\right)$ by

$$
\begin{aligned}
\boldsymbol{E}_{0}(a, x)= & \mathbf{1} \delta(1-x)+\boldsymbol{P}_{0}(x) a_{0} L+\left(\frac{1}{2} \boldsymbol{P}_{0}^{(1)}(x)+\frac{2}{3} \boldsymbol{P}_{0}(x)\right)\left(a_{0} L\right)^{2} \\
& +\left(\frac{1}{6} \boldsymbol{P}_{0}^{(2)}(x)+\frac{2}{3} \boldsymbol{P}_{0}^{(1)}(x)+\frac{16}{27} \boldsymbol{P}_{0}(x)\right)\left(a_{0} L\right)^{3} \\
& +\left(\frac{1}{24} \boldsymbol{P}_{0}^{(3)}(x)+\frac{1}{3} \boldsymbol{P}_{0}^{(2)}(x)+\frac{22}{27} \boldsymbol{P}_{0}^{(1)}(x)+\frac{16}{27} \boldsymbol{P}_{0}(x)\right)\left(a_{0} L\right)^{4} \\
& +\left(\frac{1}{120} \boldsymbol{P}_{0}^{(4)}(x)+\frac{1}{9} \boldsymbol{P}_{0}^{(3)}(x)+\frac{14}{27} \boldsymbol{P}_{0}^{(2)}(x)+\frac{80}{81} \boldsymbol{P}_{0}^{(1)}(x)+\frac{256}{405} \boldsymbol{P}_{0}(x)\right)\left(a_{0} L\right)^{5} .
\end{aligned}
$$

Here the matrices $\boldsymbol{P}_{0}^{(k)}$ are

$$
\boldsymbol{P}_{0}^{(k)}(x)=\otimes^{(k)} \boldsymbol{P}_{0}(x)
$$

i.e. $\otimes^{(1)} \boldsymbol{P}_{0}(x)=\left[\boldsymbol{P}_{0} \otimes \boldsymbol{P}_{0}\right](x)$ etc. The corresponding expressions for the $(1,1)$-components of $\boldsymbol{P}_{0}^{(k)}(x)$ are given relative to the non-singlet components $\boldsymbol{P}_{\mathrm{NS}}^{(k)}(x)$, see [15]. The singlet-matrices are

$$
\boldsymbol{P}_{0}^{(k)}(x)=\left(\begin{array}{cc}
P_{11}^{(k+1)}(x) & P_{12}^{(k+1)}(x) \\
P_{21}^{(k+1)}(x) & P_{22}^{(k+1)}(x)
\end{array}\right)
$$


with components $P_{i j}^{(k)}(x)$ given below in Eqs. (19-39). They were calculated using the convolution formulae of Appendix A and relations given in $[15,25]$ before. The projections $P_{i j}^{(k)}$ describe the splitting of a fermion into a fermion $(1,1)$, of a photon into a fermion, positron, respectively $(1,2)$, a fermion into a photon $(2,1)$, and a photon into a photon $(2,2)$ in $k$ th order in the renormalized coupling constant.

The leading order QED splitting functions can be obtained identifying $T_{R}=C_{F}=1$ and $C_{A}=0$ in the QCD splitting functions $[26,27]$ in accordance with the gauge group $U(1)$.

1st order terms:

$$
\begin{aligned}
P_{11}^{(1)}(x) & =P_{11 \mathrm{NS}}^{(1)}(x)+P_{11 \mathrm{PS}}^{(1)}(x)=\frac{2}{(1-x)_{+}}-1-x+\frac{3}{2} \delta(1-x) \\
P_{11 \mathrm{PS}}^{(1)}(x) & =0 \\
P_{12}^{(1)}(x) & =2\left[x^{2}+(1-x)^{2}\right]=4 x^{2}-4 x+2 \\
P_{21}^{(1)}(x) & =\frac{1+(1-x)^{2}}{x}=x-2+\frac{2}{x} \\
P_{22}^{(2)}(x) & =-\frac{2}{3} \delta(1-x)
\end{aligned}
$$

The $\delta(1-x)$ distribution in (23) emerges due to momentum conservation for the photon momentum $\int_{0}^{1} d x x\left[P_{12}(x)+P_{22}(x)\right]=1$.

The 2nd order terms are :

$$
\begin{aligned}
P_{11}^{(2)}(x)= & P_{11 \mathrm{NS}}^{(2)}(x)+P_{11 \mathrm{PS}}^{(2)}(x) \\
= & 8\left(\frac{\ln (1-x)}{1-x}\right)_{+}-4(1+x) \ln (1-x)+\ln (x)\left[7+7 x-\frac{4}{1-x}\right] \\
& +\frac{6}{(1-x)_{+}}-3-3 x-\frac{8}{3} x^{2}+\frac{8}{3 x}+\left[\frac{9}{4}-4 \zeta(2)\right] \delta(1-x) \\
P_{12}^{(2)}(x)= & 4\left(1-2 x+2 x^{2}\right) \ln (1-x)-2\left(1-2 x+4 x^{2}\right) \ln (x)-\frac{7}{3}+\frac{20}{3} x-\frac{8}{3} x^{2} \\
P_{21}^{(2)}(x)= & 2 \ln (1-x)\left[x-2+\frac{2}{x}\right]+(2-x) \ln (x)+\frac{10}{3}-\frac{7}{6} x-\frac{4}{3 x} \\
P_{22}^{(2)}(x)= & 4(1+x) \ln (x)+2-2 x-\frac{8}{3} x^{2}+\frac{8}{3 x}+\frac{4}{9} \delta(1-x)
\end{aligned}
$$

They were derived in [7] and various other places before.

\section{3rd order terms:}

$$
\begin{aligned}
P_{11}^{(3)}(x)= & P_{11 \mathrm{NS}}^{(3)}(x)+P_{11 \mathrm{PS}}^{(3)}(x) \\
= & 24\left(\frac{\ln ^{2}(1-x)}{1-x}\right)_{+}-12(1+x) \ln ^{2}(1-x)+36\left(\frac{\ln (1-x)}{1-x}\right)_{+} \\
& -\ln (1-x)\left[22+14 x+\frac{32}{3} x^{2}-\frac{32}{3 x}\right]-24 \ln (x) \frac{\ln (1-x)}{1-x}
\end{aligned}
$$




$$
\begin{aligned}
& +34(1+x) \ln (x) \ln (1-x)+\ln ^{2}(x)\left[\frac{4}{1-x}-\frac{15}{2}-\frac{15}{2} x\right] \\
& +\ln (x)\left[\frac{83}{6}+\frac{59}{6} x+\frac{32}{3} x^{2}-\frac{18}{1-x}\right]+22(1+x) \operatorname{Li}_{2}(1-x) \\
& +\left[\frac{27}{2}-24 \zeta(2)\right] \frac{1}{(1-x)_{+}}-\frac{317}{12}+\frac{155}{12} x+\frac{16}{9} x^{2}-\frac{16}{9 x}+12(1+x) \zeta(2) \\
& +\left[\frac{27}{8}-18 \zeta(2)+16 \zeta(3)\right] \delta(1-x) \\
& P_{12}^{(3)}(x)=8\left(1-2 x+2 x^{2}\right) \ln ^{2}(1-x)-\frac{4}{3}\left(5-16 x+4 x^{2}\right) \ln (1-x) \\
& -8\left(1-2 x+4 x^{2}\right) \ln (x) \ln (1-x)-\left(3-6 x-8 x^{2}\right) \ln ^{2}(x) \\
& -\ln (x)\left[\frac{32}{3}+\frac{26}{3} x-16 x^{2}\right]-16 x^{2} \operatorname{Li}_{2}(1-x) \\
& -\frac{521}{18}+\frac{491}{9} x-\frac{232}{9} x^{2}+\frac{32}{9 x}-8\left(1-2 x+2 x^{2}\right) \zeta(2) \\
& P_{21}^{(3)}(x)=4\left[x-2+\frac{2}{x}\right] \ln ^{2}(1-x)-\frac{2}{3}\left[5 x-16+\frac{4}{x}\right] \ln (1-x) \\
& +4(2-x) \ln (x) \ln (1-x)+\frac{3}{2}(2-x) \ln ^{2}(x)+\frac{1}{3}\left(26 x-19-\frac{16}{x}\right) \ln (x) \\
& +\frac{8}{x} \operatorname{Li}_{2}(1-x)-4\left[x-2+\frac{2}{x}\right] \zeta(2)+\frac{491}{18}-\frac{521}{36} x+\frac{16}{9} x^{2}-\frac{116}{9 x} \\
& P_{22}^{(3)}(x)=4 \ln (1-x)\left[1-x-\frac{4}{3} x^{2}+\frac{4}{3} \frac{1}{x}\right]+8(1+x) \ln (x) \ln (1-x)-2(1+x) \ln ^{2}(x) \\
& -\frac{4}{3}\left(4+x-4 x^{2}\right) \ln (x)+8(1+x) \operatorname{Li}_{2}(1-x)-\frac{31}{3}(1-x)+\frac{32}{9}\left[x^{2}-\frac{1}{x}\right] \\
& -\frac{8}{27} \delta(1-x)
\end{aligned}
$$

\section{4th order terms:}

Here and for the 5 th order terms we refer to the expressions $P_{\mathrm{NS}}^{(k)}(x)$ given in Ref. [15] for brevity.

$$
\begin{aligned}
P_{11}^{(4)}(x)= & P_{\mathrm{NS}}^{(4)}(x)+48(1+x) \ln (x) \ln ^{2}(1-x)-24(1+x) \ln ^{2}(x) \ln (1-x) \\
& -\frac{2}{3}(1+x) \ln ^{3}(x)+8\left[3-3 x-4 x^{2}+\frac{4}{x}\right] \ln ^{2}(1-x) \\
& -8 \ln (x) \ln (1-x)\left[\frac{4}{3}-\frac{14}{3} x-8 x^{2}\right]+\ln ^{2}(x)\left[-\frac{25}{3}+\frac{5}{3} x-16 x^{2}\right] \\
& -4\left[\frac{73}{3}-\frac{73}{3} x-\frac{16}{9} x^{2}+\frac{16}{9 x}\right] \ln (1-x) \\
& -\left[\frac{569}{9}+\frac{1445}{9} x+\frac{128}{9} x^{2}+\frac{64}{9 x}+48 \zeta(2)+48 \zeta(2) x\right] \ln (x) \\
& +96(1+x) \ln (1-x) \operatorname{Li}_{2}(1-x)+8\left[\frac{5}{3}+\frac{5}{3} x+4 x^{2}+\frac{4}{x}\right] \operatorname{Li}_{2}(1-x) \\
& -96(1+x) \operatorname{Li}_{3}(1-x)+48(1+x) \mathrm{S}_{1,2}(1-x)-\frac{745}{18}+\frac{745}{18} x+\frac{224}{9} x^{2}-\frac{224}{9 x}
\end{aligned}
$$




$$
\begin{aligned}
& -8\left[3-3 x-4 x^{2}+\frac{4}{x}\right] \zeta(2) \\
& P_{12}^{(4)}=16\left(1-2 x+2 x^{2}\right) \ln ^{3}(1-x)-24\left(1-2 x+4 x^{2}\right) \ln (x) \ln ^{2}(1-x) \\
& -2 \ln ^{2}(x) \ln (1-x)\left(5-10 x-24 x^{2}\right)+\ln ^{3}(x)\left[\frac{7}{3}-\frac{14}{3} x-\frac{16}{3} x^{2}\right] \\
& -4 \ln ^{2}(1-x)\left[\frac{13}{3}-\frac{44}{3} x+\frac{8}{3} x^{2}\right]-4 \ln (x) \ln (1-x)\left[\frac{32}{3}+\frac{35}{3} x-16 x^{2}\right] \\
& +\ln ^{2}(x)\left[\frac{79}{6}+\frac{14}{3} x-\frac{80}{3} x^{2}\right] \\
& -\ln (1-x)\left[\frac{959}{9}-\frac{1978}{9} x+\frac{320}{3} x^{2}-\frac{128}{9 x}+48 \zeta(2)-96 \zeta(2) x+96 \zeta(2) x^{2}\right] \\
& +\ln (x)\left[\frac{1505}{18}-\frac{614}{9} x+\frac{832}{9} x^{2}+24 \zeta(2)-48 \zeta(2) x+96 \zeta(2) x^{2}\right] \\
& -96 x^{2} \ln (1-x) \operatorname{Li}_{2}(1-x)-44(1-2 x) \ln (x) \operatorname{Li}_{2}(1-x) \\
& -4 \mathrm{Li}_{2}(1-x)\left[15-3 x-\frac{40}{3} x^{2}\right]+96 x^{2} \operatorname{Li}_{3}(1-x) \\
& -4\left(13-26 x+16 x^{2}\right) \mathrm{S}_{1,2}(1-x)+\frac{18065}{108}-\frac{5947}{27} x+\frac{560}{9} x^{2}-\frac{128}{27 x} \\
& +4\left[\frac{13}{3}-\frac{44}{3}+\frac{8}{3} x^{2}\right] \zeta(2)+32\left(1-2 x+2 x^{2}\right) \zeta(3) \\
& P_{21}^{(4)}=8\left[x-2+\frac{2}{x}\right] \ln ^{3}(1-x)+12(2-x) \ln (x) \ln ^{2}(1-x) \\
& +5(2-x) \ln ^{2}(x) \ln (1-x)-\frac{7}{6}(2-x) \ln ^{3}(x)+\frac{2}{3} \ln ^{2}(1-x)\left[44-13 x-\frac{8}{x}\right] \\
& -2 \ln (x) \ln (1-x)\left[\frac{53}{3}-\frac{58}{3} x+\frac{32}{3} \frac{1}{x}\right]+\ln ^{2}(x)\left[\frac{16}{3}-\frac{101}{12} x\right] \\
& +\ln (1-x)\left[\frac{989}{9}-\frac{959}{18} x+\frac{64}{9} x^{2}-\frac{160}{3 x}+48 \zeta(2)-24 \zeta(2) x-\frac{48}{x} \zeta(2)\right] \\
& +\ln (x)\left[-\frac{682}{9}+\frac{413}{36} x-\frac{64}{9} x^{2}+\frac{64}{9 x}-24 \zeta(2)+12 \zeta(2) x\right] \\
& +\frac{48}{x} \ln (1-x) \operatorname{Li}_{2}(1-x)+22(2-x) \ln (x) \operatorname{Li}_{2}(1-x) \\
& -2 \operatorname{Li}_{2}(1-x)\left[3-15 x+\frac{40}{3 x}\right]-\frac{48}{x} \operatorname{Li}_{3}(1-x)+2\left[26-13 x+\frac{8}{x}\right] \mathrm{S}_{1,2}(1-x) \\
& -\frac{5947}{54}+\frac{18065}{216} x-\frac{64}{27} x^{2}+\frac{280}{9 x}-\frac{2}{3}\left[44-13 x-\frac{8}{x}\right] \zeta(2) \\
& -16\left[2-x-\frac{2}{x}\right] \zeta(3) \\
& P_{22}^{(4)}(x)=16(1+x) \ln (x) \ln ^{2}(1-x)-8(1+x) \ln ^{2}(x) \ln (1-x)-2(1+x) \ln ^{3}(x) \\
& +8\left[1-x-\frac{4}{3} x^{2}+\frac{4}{3 x}\right] \ln ^{2}(1-x)-\frac{16}{3}\left(2-x-4 x^{2}\right) \ln (x) \ln (1-x)
\end{aligned}
$$




$$
\begin{aligned}
& -\frac{1}{3}\left(19-23 x+16 x^{2}\right) \ln ^{2}(x)-4 \ln (1-x)\left[9-9 x-\frac{16}{9} x^{2}+\frac{16}{9 x}\right] \\
& -\ln (x)\left[\frac{217}{3}+\frac{325}{3} x+\frac{128}{9} x^{2}+\frac{64}{9 x}+16 \zeta(2)+16 \zeta(2) x\right] \\
& +32(1+x) \ln (1-x) \operatorname{Li}_{2}(1-x)-\frac{8}{3} \operatorname{Li}_{2}(1-x)\left[1+x-4 x^{2}-\frac{4}{x}\right] \\
& -32(1+x) \operatorname{Li}_{3}(1-x)+16(1+x) \mathrm{S}_{1,2}(1-x) \\
& -\frac{1331}{18}+\frac{1331}{18} x+\frac{608}{27} x^{2}-\frac{608}{27 x}-8\left[1-x-\frac{4}{3} x^{2}+\frac{4}{3 x}\right] \zeta(2) \\
& +\frac{16}{81} \delta(1-x)
\end{aligned}
$$

\section{5th order terms:}

$$
\begin{aligned}
& P_{11}^{(5)}(x)=P_{\mathrm{NS}}^{(5)}(x)+128(1+x) \ln (x) \ln ^{3}(1-x)-96(1+x) \ln ^{2}(x) \ln ^{2}(1-x) \\
& +\frac{4}{3}(1+x) \ln ^{4}(x)+64\left[1-x-\frac{4}{3} x^{2}+\frac{4}{3 x}\right] \ln ^{3}(1-x) \\
& -32\left(1-5 x-8 x^{2}\right) \ln (x) \ln ^{2}(1-x)-8\left(7+x+16 x^{2}\right) \ln ^{2}(x) \ln (1-x) \\
& +\frac{8}{9}\left[10-5 x+16 x^{2}\right] \ln ^{3}(x)-64\left[6-6 x-\frac{x^{2}}{3}+\frac{1}{3 x}\right] \ln ^{2}(1-x) \\
& -32 \ln (x) \ln (1-x)\left[\frac{97}{9}+\frac{313}{9} x+\frac{8}{3} x^{2}+\frac{4}{3 x}+12 \zeta(2)+12 \zeta(2) x\right] \\
& +16 \ln ^{2}(x)\left[\frac{83}{9}+\frac{122}{9} x+2 x^{2}+6 \zeta(2)+6 \zeta(2) x\right] \\
& -4 \ln (1-x)\left[\frac{331}{9}-\frac{331}{9} x-\frac{1024}{27} x^{2}+\frac{1024}{27 x}\right. \\
& \left.+48 \zeta(2)-48 \zeta(2) x-64 \zeta(2) x^{2}+\frac{64}{x} \zeta(2)\right] \\
& +4 \ln (x)\left[\frac{1990}{27}+\frac{997}{27} x-\frac{320}{9} x^{2}+\frac{64}{27 x}\right. \\
& \left.+8 \zeta(2)-40 \zeta(2) x-64 \zeta(2) x^{2}+64 \zeta(3)+64 \zeta(3) x\right] \\
& +384(1+x) \ln ^{2}(1-x) \operatorname{Li}_{2}(1-x)-96(1+x) \ln ^{2}(x) \operatorname{Li}_{2}(1-x) \\
& +128\left[1+x+2 x^{2}+\frac{2}{x}\right] \ln (1-x) \operatorname{Li}_{2}(1-x) \\
& -144(1-x) \ln (x) \operatorname{Li}_{2}(1-x)-768(1+x) \ln (1-x) \operatorname{Li}_{3}(1-x) \\
& +384(1+x) \ln (1-x) \mathrm{S}_{1,2}(1-x)-256(1+x) \ln (x) \mathrm{S}_{1,2}(1-x) \\
& -32\left[\frac{205}{9}+\frac{205}{9} x+2 x^{2}+\frac{2}{x}+12 \zeta(2)+12 \zeta(2) x\right] \operatorname{Li}_{2}(1-x) \\
& -128\left[1+x+2 x^{2}+\frac{2}{x}\right] \operatorname{Li}_{3}(1-x)-16\left[7-15 x-\frac{32}{3} x^{2}-\frac{16}{3 x}\right] \mathrm{S}_{1,2}(1-x) \\
& +768(1+x) \operatorname{Li}_{4}(1-x)-192(1+x) \mathrm{S}_{1,3}(1-x)-384(1+x) \mathrm{S}_{2,2}(1-x)
\end{aligned}
$$




$$
\begin{aligned}
& +\frac{2}{27}\left[10127-10127 x-\frac{2456}{3} x^{2}+\frac{2456}{3 x}\right]+64\left[6-6 x-\frac{1}{3} x^{2}+\frac{1}{3 x}\right] \zeta(2) \\
& +128\left[1-x-\frac{4}{3} x^{2}+\frac{4}{3 x}\right] \zeta(3) \\
& P_{12}^{(5)}(x)=32\left(1-2 x+2 x^{2}\right) \ln ^{4}(1-x)-64\left(1-2 x+4 x^{2}\right) \ln (x) \ln ^{3}(1-x) \\
& -24\left(1-2 x-8 x^{2}\right) \ln ^{2}(x) \ln ^{2}(1-x)+\frac{8}{3}\left(5-10 x-16 x^{2}\right) \ln ^{3}(x) \ln (1-x) \\
& +\ln ^{4}(x)\left[\frac{5}{12}-\frac{5}{6} x+\frac{8}{3} x^{2}\right]-\frac{64}{3}\left(2-7 x+x^{2}\right) \ln ^{3}(1-x) \\
& -16\left(8+11 x-12 x^{2}\right) \ln (x) \ln ^{2}(1-x)+\frac{8}{3}\left(26+23 x-60 x^{2}\right) \ln ^{2}(x) \ln (1-x) \\
& +\ln ^{3}(x)\left[5-\frac{x}{3}+\frac{224}{9} x^{2}\right] \\
& -4 \ln ^{2}(1-x)\left[\frac{649}{9}-\frac{1478}{9} x+\frac{728}{9} x^{2}-\frac{32}{3 x}\right. \\
& \left.+48 \zeta(2)-96 \zeta(2) x+96 \zeta(2) x^{2}\right] \\
& +4 \ln (x) \ln (1-x)\left[\frac{1027}{9}-\frac{866}{9} x+\frac{1328}{9} x^{2}+48 \zeta(2)-96 \zeta(2) x+192 \zeta(2) x^{2}\right] \\
& +\ln ^{2}(x)\left[\frac{451}{9}-\frac{1664}{9} x-\frac{1072}{9} x^{2}+24 \zeta(2)-48 \zeta(2) x-192 \zeta(2) x^{2}\right] \\
& +4 \ln (1-x)\left[\frac{6064}{27}-\frac{7721}{27} x+\frac{2056}{27} x^{2}-\frac{128}{27 x}+32 \zeta(2)-112 \zeta(2) x+16 \zeta(2) x^{2}\right. \\
& \left.+64 \zeta(3)-128 \zeta(3) x+128 \zeta(3) x^{2}\right] \\
& +\ln (x)\left[\frac{8861}{54}+\frac{24511}{27} x-416 x^{2}-\frac{256}{27 x}+128 \zeta(2)+176 \zeta(2) x-192 \zeta(2) x^{2}\right. \\
& \left.-128 \zeta(3)+256 \zeta(3) x-512 \zeta(3) x^{2}\right] \\
& -384 x^{2} \ln ^{2}(1-x) \operatorname{Li}_{2}(1-x)+288(2 x-1) \ln (x) \ln (1-x) \operatorname{Li}_{2}(1-x) \\
& +16\left(1-2 x+4 x^{2}\right) \ln ^{2}(x) \operatorname{Li}_{2}(1-x)-32\left(12-3 x-10 x^{2}\right) \ln (1-x) \operatorname{Li}_{2}(1-x) \\
& +\frac{32}{3}\left(1-5 x-12 x^{2}\right) \ln (x) \operatorname{Li}_{2}(1-x)+768 x^{2} \ln (1-x) \operatorname{Li}_{3}(1-x) \\
& -288(2 x-1) \ln (x) \operatorname{Li}_{3}(1-x)-32\left(11-22 x+16 x^{2}\right) \ln (1-x) \mathrm{S}_{1,2}(1-x) \\
& -16\left(5-10 x-16 x^{2}\right) \ln (x) \mathrm{S}_{1,2}(1-x) \\
& +8\left[21+34 x+\frac{100}{3} x^{2}+\frac{16}{3 x}+48 \zeta(2) x^{2}\right] \operatorname{Li}_{2}(1-x) \\
& +32\left(12-3 x-10 x^{2}\right) \operatorname{Li}_{3}(1-x)-16\left[10+5 x-\frac{8}{3} x^{2}\right] \mathrm{S}_{1,2}(1-x) \\
& -768 x^{2} \mathrm{Li}_{4}(1-x)+32\left(1-2 x+2 x^{2}\right) \mathrm{Li}_{2}^{2}(1-x) \\
& -32\left(7-14 x-6 x^{2}\right) \mathrm{S}_{1,3}(1-x)+32\left(7-14 x+8 x^{2}\right) \mathrm{S}_{2,2}(1-x) \\
& +\frac{322519}{648}-\frac{240997}{324} x+\frac{23776}{81} x^{2}-\frac{1088}{27 x}+\frac{4}{3}\left[\frac{649}{3}-\frac{1478}{3} x+\frac{728}{3} x^{2}-\frac{32}{x}\right] \zeta(2) \\
& -\frac{128}{3}\left(2-7 x+x^{2}\right) \zeta(3)+48\left(1-2 x+2 x^{2}\right) \zeta(4)
\end{aligned}
$$




$$
\begin{aligned}
& P_{21}^{(5)}(x)=-16\left[2-x-\frac{2}{x}\right] \ln ^{4}(1-x)+32(2-x) \ln (x) \ln ^{3}(1-x) \\
& +12(2-x) \ln ^{2}(x) \ln ^{2}(1-x)-\frac{20}{3}(2-x) \ln ^{3}(x) \ln (1-x)-\frac{5}{24}(2-x) \ln ^{4}(x) \\
& +\frac{32}{3}\left[7-2 x-\frac{1}{x}\right] \ln ^{3}(1-x)-8\left[17-16 x+\frac{8}{x}\right] \ln (x) \ln ^{2}(1-x) \\
& +\frac{4}{3}(41-46 x) \ln ^{2}(x) \ln (1-x)+\frac{1}{6}(x-47) \ln ^{3}(x) \\
& +\ln ^{2}(1-x)\left[\frac{2956}{9}-\frac{1298}{9} x+\frac{64}{3} x^{2}-\frac{1456}{9 x}+192 \zeta(2)-96 \zeta(2) x-\frac{192}{x} \zeta(2)\right] \\
& -\ln (x) \ln (1-x)\left[\frac{4180}{9}-\frac{542}{9} x+\frac{128}{3} x^{2}-\frac{256}{9 x}+192 \zeta(2)-96 \zeta(2) x\right] \\
& -\ln ^{2}(x)\left[\frac{220}{9}-\frac{1207}{18} x-\frac{32}{3} x^{2}-\frac{64}{9 x}+24 \zeta(2)-12 \zeta(2) x\right] \\
& -\ln (1-x)\left[\frac{15442}{27}-\frac{12128}{27} x+\frac{256}{27} x^{2}-\frac{4112}{27 x}+224 \zeta(2)-64 \zeta(2) x-\frac{32}{x} \zeta(2)\right. \\
& \left.+256 \zeta(3)-128 \zeta(3) x-\frac{256}{x} \zeta(3)\right] \\
& +\ln (x)\left[\frac{6373}{54}-\frac{57373}{108} x+\frac{128}{9} x^{2}+\frac{1504}{27 x}+136 \zeta(2)-128 \zeta(2) x+\frac{64}{x} \zeta(2)\right. \\
& +128 \zeta(3)-64 \zeta(3) x] \\
& +\frac{192}{x} \ln ^{2}(1-x) \operatorname{Li}_{2}(1-x)+144(2-x) \ln (x) \ln (1-x) \operatorname{Li}_{2}(1-x) \\
& -8(2-x) \ln ^{2}(x) \operatorname{Li}_{2}(1-x)-16\left[3-12 x+\frac{10}{x}\right] \ln (1-x) \operatorname{Li}_{2}(1-x) \\
& -\frac{16}{3}\left[5-x+\frac{12}{x}\right] \ln (x) \operatorname{Li}_{2}(1-x)-\frac{384}{x} \ln (1-x) \operatorname{Li}_{3}(1-x) \\
& -144(2-x) \ln (x) \operatorname{Li}_{3}(1-x)+16\left[22-11 x+\frac{8}{x}\right] \ln (1-x) \mathrm{S}_{1,2}(1-x) \\
& +40(2-x) \ln (x) \mathrm{S}_{1,2}(1-x)-4\left[34+21 x+\frac{16}{3} x^{2}+\frac{100}{3 x}+\frac{48}{x} \zeta(2)\right] \operatorname{Li}_{2}(1-x) \\
& +16\left[3-12 x+\frac{10}{x}\right] \operatorname{Li}_{3}(1-x)-8\left[11-14 x+\frac{52}{3 x}\right] \mathrm{S}_{1,2}(1-x) \\
& +\frac{384}{x} \operatorname{Li}_{4}(1-x)-16\left[2-x-\frac{2}{x}\right] \operatorname{Li}_{2}^{2}(1-x) \\
& +16\left[14-7 x+\frac{2}{x}\right] \mathrm{S}_{1,3}(1-x)-16\left[14-7 x+\frac{16}{x}\right] \mathrm{S}_{2,2}(1-x) \\
& -\frac{240997}{648}+\frac{322519}{1296} x-\frac{544}{27} x^{2}+\frac{11888}{81 x}-\left[\frac{2956}{9}-\frac{1298}{9} x+\frac{64}{3} x^{2}-\frac{1456}{9 x}\right] \zeta(2) \\
& +\frac{64}{3}\left[7-2 x-\frac{1}{x}\right] \zeta(3)-24\left[2-x-\frac{2}{x}\right] \zeta(4) \\
& P_{22}^{(5)}(x)=32(1+x) \ln (x) \ln ^{3}(1-x)-24(1+x) \ln ^{2}(x) \ln ^{2}(1-x)
\end{aligned}
$$




$$
\begin{aligned}
& -\frac{20}{3}(1+x) \ln ^{3}(x) \ln (1-x)+\frac{7}{6}(1+x) \ln ^{4}(x) \\
& +16\left[1-x-\frac{4}{3} x^{2}+\frac{4}{3 x}\right] \ln ^{3}(1-x)-\frac{16}{3}\left(4-5 x+12 x^{2}\right) \ln (x) \ln ^{2}(1-x) \\
& -\ln ^{2}(x) \ln (1-x)\left[\frac{82}{3}-\frac{74}{3} x+32 x^{2}\right]+\frac{2}{9} \ln ^{3}(x)\left[35-10 x+16 x^{2}\right] \\
& -\frac{4}{3}\left[77-77 x-\frac{32}{3} x^{2}+\frac{32}{3 x}\right] \ln ^{2}(1-x) \\
& -\ln (x) \ln (1-x)\left[\frac{862}{3}+\frac{1478}{3} x+\frac{512}{9} x^{2}+\frac{256}{9 x}+96 \zeta(2)+96 \zeta(2) x\right] \\
& +\ln ^{2}(x)\left[\frac{319}{3}+\frac{266}{3} x+\frac{64}{3} x^{2}+24 \zeta(2)+24 \zeta(2) x\right] \\
& -\ln (1-x)\left[\frac{2401}{9}-\frac{2401}{9} x-\frac{2624}{27} x^{2}+\frac{2624}{27 x}\right. \\
& \left.+48 \zeta(2)-48 \zeta(2) x-64 \zeta(2) x^{2}+\frac{64}{x} \zeta(2)\right] \\
& +\ln (x)\left[\frac{8680}{27}+\frac{1477}{27} x-\frac{2240}{27} x^{2}+\frac{128}{9 x}\right. \\
& \left.+\frac{64}{3} \zeta(2)-\frac{80}{3} \zeta(2) x-64 \zeta(2) x^{2}+64 \zeta(3)+64 \zeta(3) x\right] \\
& +96(1+x) \ln ^{2}(1-x) \operatorname{Li}_{2}(1-x)-44(1+x) \ln ^{2}(x) \operatorname{Li}_{2}(1-x) \\
& +\frac{16}{3}\left[1+x+12 x^{2}+\frac{12}{x}\right] \ln (1-x) \operatorname{Li}_{2}(1-x)-76(1-x) \ln (x) \operatorname{Li}_{2}(1-x) \\
& -192(1+x) \ln (1-x) \operatorname{Li}_{3}(1-x)+96(1+x) \ln (1-x) \mathrm{S}_{1,2}(1-x) \\
& -104(1+x) \ln (x) \mathrm{S}_{1,2}(1-x) \\
& -\mathrm{Li}_{2}(1-x)\left[390+390 x+\frac{128}{3} x^{2}+\frac{128}{3 x}+96 \zeta(2)+96 \zeta(2) x\right] \\
& -\frac{16}{3}\left[1+x+12 x^{2}+\frac{12}{x}\right] \operatorname{Li}_{3}(1-x)-\frac{4}{3}\left[61-65 x-32 x^{2}-\frac{16}{x}\right] \mathrm{S}_{1,2}(1-x) \\
& +192(1+x) \operatorname{Li}_{4}(1-x)-88(1+x) \mathrm{S}_{1,3}(1-x)-96(1+x) \mathrm{S}_{2,2}(1-x) \\
& +\frac{24043}{36}-\frac{24043}{36} x-\frac{5408}{81} x^{2}+\frac{5408}{81 x}+\frac{4}{3}\left[77-77 x-\frac{32}{3} x^{2}+\frac{32}{3 x}\right] \zeta(2) \\
& +32\left[1-x-\frac{4}{3} x^{2}+\frac{4}{3 x}\right] \zeta(3)-\frac{32}{243} \delta(1-x)
\end{aligned}
$$

The complexity of the above expressions reaches weight $w=n+p=4$ Nielsen integrals

$$
S_{n, p}(x)=\frac{(-1)^{n+p-1}}{(n-1) ! p !} \int_{0}^{1} \frac{d z}{z} \ln ^{n-1}(z) \ln ^{p}(1-z x) .
$$

The radiators can be expressed through these functions and polynomials thereof as well as rational functions in $x$.

The universal radiator functions Eq. (14) can now be attached to the respective initial- or final-state radiating light charged fermion or photon lines of any differential scattering cross section to account for the respective leading order QED corrections. These radiators generalize the 
radiators due to soft-photon exponentiation, valid for $D_{\mathrm{NS}}\left(a\left(Q^{2}\right), x\right)[29]$ in the region $x \rightarrow 1$, to general values of $x$ and all collinear transitions possible. The numerical effect of the respective radiator depends on the change of the subsystem kinematics of the differential scattering cross section, which usually differs for initial and final state radiation and due to the type of leg encountered. This kinematics has to be worked out for the respective process accordingly. Moreover, the radiative corrections may strongly depend on the way the kinematic variables of the process are measured. In case of deeply inelastic scattering investigations of these aspects were performed in $[3,8,9,28]$. For similar considerations for $e^{+} e^{-}$annihilation see e.g. Ref. [2].

The radiative correction due to the radiator $D_{a_{1} a_{2}}\left(a\left(Q^{2}\right), x\right)$ for a differential cross section reads

$$
\frac{d^{l} \sigma_{a_{1}}}{d b_{1} \ldots d b_{l}}=\left.\int_{0}^{1} d z D_{a_{1} a_{2}}\left(a\left(Q^{2}\right), z\right) \theta\left(z-z_{0}^{a_{1}}\right) J^{a_{1}}\left(\left.b_{r}\right|_{r=1} ^{l}, z\right) \frac{d^{l} \sigma_{a_{2}}}{d b_{1} \ldots d b_{l}}\right|_{b_{1}=\hat{b}_{1} \ldots b_{l}=\hat{b}_{1}} .
$$

Here the $l$ kinematic variables which determine the differential cross section are $\left.b_{r}\right|_{r=1} ^{l}$. Their rescaled value under changing the momentum $p_{a_{1}} \rightarrow z \cdot p_{a_{1}}$ resp. $p_{a_{1}} \rightarrow p_{a_{1}} / z$ for initial or final state radiation, $\left.\hat{b}_{r}\right|_{r=1} ^{l}$, is bounded by $z_{0}^{a_{1}}$ for hard radiation. $J^{a_{1}}\left(\left.b_{r}\right|_{r=1} ^{l}, z\right)$ denotes the corresponding Jacobian

$$
J^{a_{1}}\left(\left.b_{r}\right|_{r=1} ^{l}, z\right)=\left|\begin{array}{ccc}
\partial \hat{b}_{1} / \partial b_{1} & \ldots & \partial \hat{b}_{l} / \partial b_{1} \\
\vdots & & \vdots \\
\partial \hat{b}_{l} / \partial b_{1} & \ldots & \partial \hat{b}_{l} / \partial b_{l}
\end{array}\right|
$$

and $d^{l} \sigma_{a_{2}} / d b_{1} \ldots d b_{l}$ is the subsystem differential cross section for which the line of type $a_{1}$ is being replaced by a line of type $a_{2}$. Eq. (41) may be generalized to the case of more universal radiators correspondingly, requiring additional rescaling of variables.

In the above we assumed, that the radiator functions describe collinear radiation along outer fermions or photons. However, in various applications also internal, nearly collinear situations may occur. One example is the so-called 3rd [30] or Compton peak [2,3,31] in deeply inelastic scattering. Here a photon being originally virtual contributes near to its mass shell in the radiative correction, which gives rise to a factorizing collinear process. The universal contributions to these processes can be obtained from radiators as well.

Finally we would like to add a remark on small $x$ resummations. In QCD the leading order corrections stem from

$$
\begin{aligned}
P_{g g}^{x \rightarrow 0}(x) & =\boldsymbol{M}^{-1}\left[\gamma_{L}\left(N, a_{s}\right)\right](x) \\
P_{g q}^{x \rightarrow 0}(x) & =\frac{C_{F}}{C_{A}} P_{g g}^{x \rightarrow 0}(x)
\end{aligned}
$$

and the function $\gamma_{L}\left(N, a_{s}\right)$ obeys

$$
\gamma_{L}\left(N, a_{s}\right)=\frac{C_{A} a_{s}}{\pi(N-1)}\left\{1+2 \sum_{l=1}^{\infty} \zeta_{2 l+1} \gamma_{L}^{2 l+1}\right\}
$$

with $a_{s}=\alpha_{s} /(4 \pi)$ the strong coupling constant and $\zeta_{k}$ Riemann's $\zeta$-function. The transition to QED, $C_{A} \rightarrow 0, C_{F} \rightarrow 1$ trivializes $(43,44)$ except for the lowest order term in $a\left(Q^{2}\right)$ in $P_{\gamma f}^{x \rightarrow 0}(x)$ which is already known from the fixed order terms above. Yet all anomalous dimensions do 
receive $1 / x$ terms in higher orders, which contribute, as well-known [32], in the abelian limit starting from next-to-leading order :

$$
\begin{aligned}
P_{f f}^{(1)}(x) & \propto \frac{1}{x} \frac{40}{9} N_{f} \\
P_{\gamma f}^{(1)}(x) & \propto \frac{1}{x} \frac{40}{9} N_{f} \\
P_{f \gamma}^{(1)}(x) & \propto \frac{1}{x} \frac{40}{9} N_{f} \\
P_{\gamma \gamma}^{(1)}(x) & \propto \frac{1}{x} \frac{4}{3} N_{f} .
\end{aligned}
$$

However, these terms do not stem from the resummation [19] but are of different origin. Their pole strength is of $O\left(\alpha^{2} /(N-1)\right)$, which is larger than for the poles resulting from $O\left(\alpha^{2} \ln ^{2}(x)\right)$ terms. For unpolarized QED radiators systematic resummations of the leading small $x$ terms were not carried out yet. At leading order in $a\left(Q^{2}\right)$ only $P_{\gamma f}(x) \propto 1 / x$, while at next-toleading order (46-49) all terms contain a singular contribution. It would be worthwhile to derive resummations of these terms in the future.

\section{Numerical Results}

The singlet contributions to the universal radiator functions, summing the leading logarithmic corrections up to $O\left((\alpha L)^{5}\right)$, are shown in Figure 1, as a function of the momentum fraction $x$ for different values of $Q=\sqrt{Q^{2}}$ in the case of $m_{f}=m_{e}$. The corrections in case of other charged fermions have to be rescaled accordingly in $L=\ln \left(Q^{2} / m_{f}^{2}\right)$. In case of fractionally charged leptons, $\alpha_{0}$ has to be replaced by $e_{f}^{2} \alpha_{0}$. Here $D_{11}^{P S}$ denotes the pure singlet part of the fermion radiator to which the non-singlet contribution has to be added, cf. [15]. The diagonal radiator $D_{11}^{\mathrm{PS}}$ vanishes at leading order $O(\alpha L)$, while the radiator $D_{22}$ contributes at $x=1$ only due to momentum conservation. For $x<1$ the (pure) singlet $(P S)$-diagonal radiators contribute with $O\left((\alpha L)^{2}\right)$ only. In this order the $P S$-diagonal terms are identical in the region $x<1$ and differ by the $\delta(1-x)$-distribution in $D_{22}$. At higher orders both diagonal elements receive different corrections. This explains the relative smallness of the radiators $D_{11}^{\mathrm{PS}}$ and $D_{22}$ compared to $D_{12}$ and $D_{21}$. Yet the diagonal radiators grow $\propto 1 / x$ as $x \rightarrow 0$. This growth is even more pronounced in $D_{21}$, which contains a $1 / x$ term already at $O(\alpha L)$, while $D_{12}$ receives those terms at $O\left((\alpha L)^{3}\right)$ only and therefore shows a moderately varying profile in $x$. The QED scaling violations shown in Figure 1 are of moderate size, comparing scales from $Q=10 \mathrm{GeV}$ to $Q=1 \mathrm{TeV}$ which is due to the smallness of the fine structure constant and its weak running. At $x=0.1$ the radiators $D_{11}^{\mathrm{PS}}$ and $D_{22}$ reach about $1 \%$ and grow further towards smaller values of $x . D_{12}$ takes values between 3 and $8 \% . D_{21}$ is largest and reaches $50 \%$ at $x=0.1$. The radiators $D_{11}^{\mathrm{PS}}, D_{21}$ and $D_{22}$ vanish towards $x \rightarrow 1$, while $D_{12}$ approaches finite values.

Figure 2 compares the size of the first order contribution to $D_{11}, D_{12}$ and $D_{21}$ with the respective contributions up to $O\left((\alpha L)^{5}\right)$, which can be regarded numerically as the total contribution for the values of $Q$ chosen. For $D_{11}$ the first order contribution is much smaller than the total contribution in the region of small values $x$ due to the steep rise of the pure singlet component. The higher order contributions to $D_{12}$ are small at medium values of $x$, and amount to a $-5 \%$ correction in the small $x$ region and a $+10 \%$ correction at large values of $x$ for $Q=10 \mathrm{GeV}$. The higher order contribution to $D_{21}$ range between $1 \%$ to $10 \%$. 
Figure 3 shows the impact of the 5 th order term w.r.t. the first four orders. The effect of the 5th order term amounts to $O\left(10^{-5}\right)$ for $D_{11}, D_{12}$ and $D_{21}$, while the effect in the case of $D_{22}$ is one order of magnitude larger at large value of $x$. In either case, we confirm that the singlet radiator to the 5th order has a very high accuracy, which is sufficient to represent the universal part of QED corrections, relevant in high precision measurements in both high-energy charged lepton anti-lepton collision, charged lepton-nucleon collisions and photon collisions at future electron-positron linear colliders with a possible Giga- $Z$ option, electron-photon and photonphoton colliders and future muon colliders. These are the reactions to which the corresponding radiators are contributing. The reactions do also contribute to the precision measurements of QCD scaling violations $[3,28,33]$ in deeply inelastic scattering as universal QED corrections. Likewise they are important for rare initial states at high energy hadron colliders such as LHC and would contribute in lepton and photon initiated processes there, such as single leptoquark production [34].

The radiator functions calculated above are made available in form of a FORTRAN-program which can be obtained form the authors upon request.

\section{Conclusions}

The collinear logarithms in QED can be resummed due to the renormalization group equations for mass factorization. Unlike the case in QCD the collinear logarithms are finite due to the possibility to define the coupling constant asymptotically, i.e. in the limit of vanishing scales. The associated logarithms are well defined since photons and leptons are non-confined and may be regarded as stable or long-lived states. The leading order corrections $O\left((\alpha L)^{k}\right)$ are universal. The respective radiators resum the radiative corrections which only depend on the type of particle transition $i \rightarrow j$. As shown, sufficient numerical stability of $O\left(10^{-4 \ldots-5}\right)$ is reached evaluating the radiators to $O\left((\alpha L)^{5}\right)$ for scales as large as $Q \lesssim 1 \mathrm{TeV}$. The radiators are presented in $x$-space and can be applied directly to the respective multiply differential scattering cross sections to describe the universal contribution due to initial and final state radiation off the different outer legs contributing to the respective scattering process involving charged fermions and photons. In the small $x$ region the leading order radiators receive contributions $\propto 1 / x$, with an onset in different orders in $\alpha L$, which leads to larger corrections in this kinematic region. A systematic resummation of the particular small $x$ contributions, unlike the case for the non-singlet and polarized singlet corrections, is not known yet. The radiators derived can easily be adopted for experimental analysis and simulation programs. 


\section{Figures}

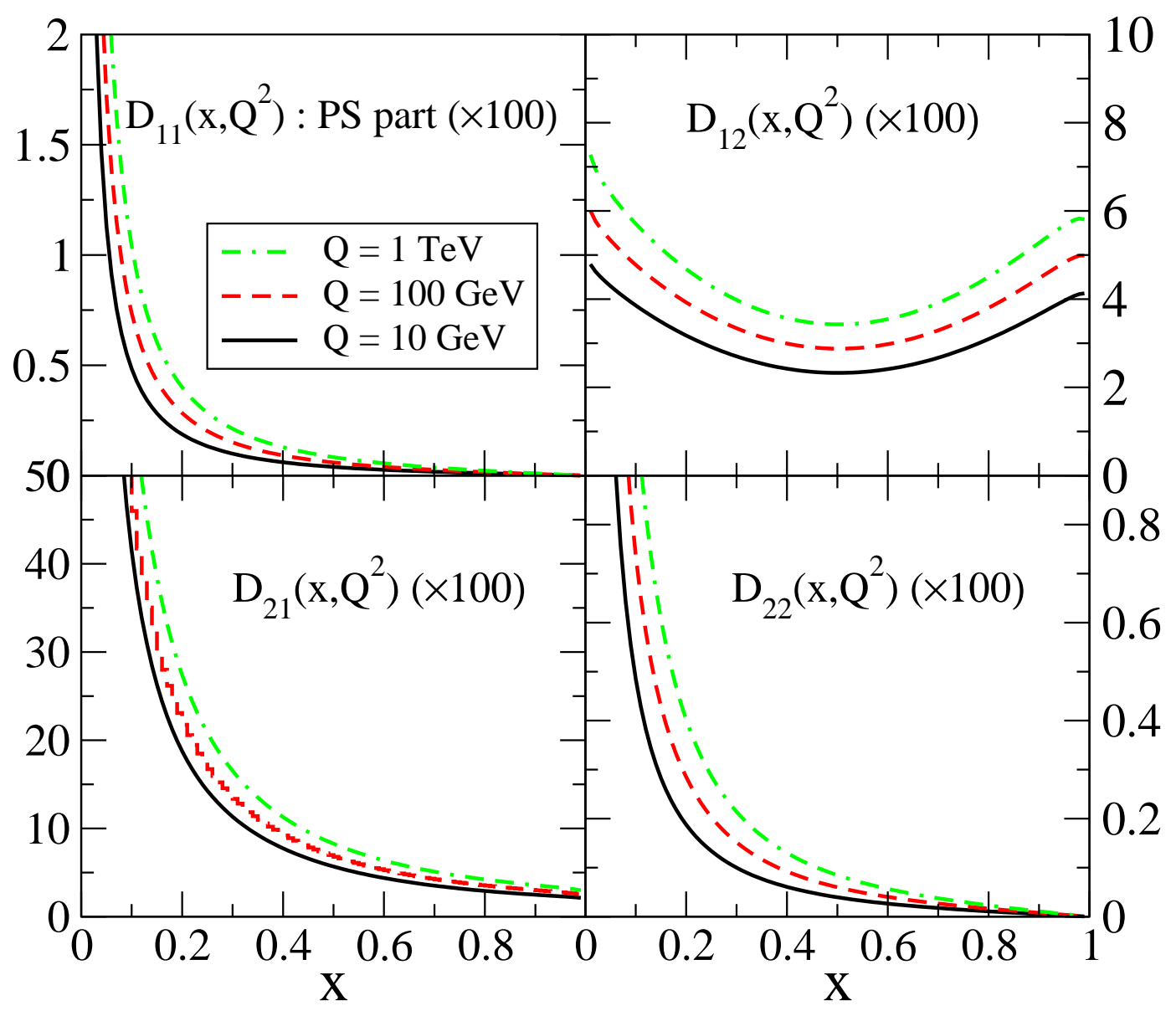

Figure 1: The singlet radiators $D_{i j}$ as a function of $x$ and $Q$ in $\% . D_{11}^{P S}$ denotes the pure singlet part of $D_{11}$. 


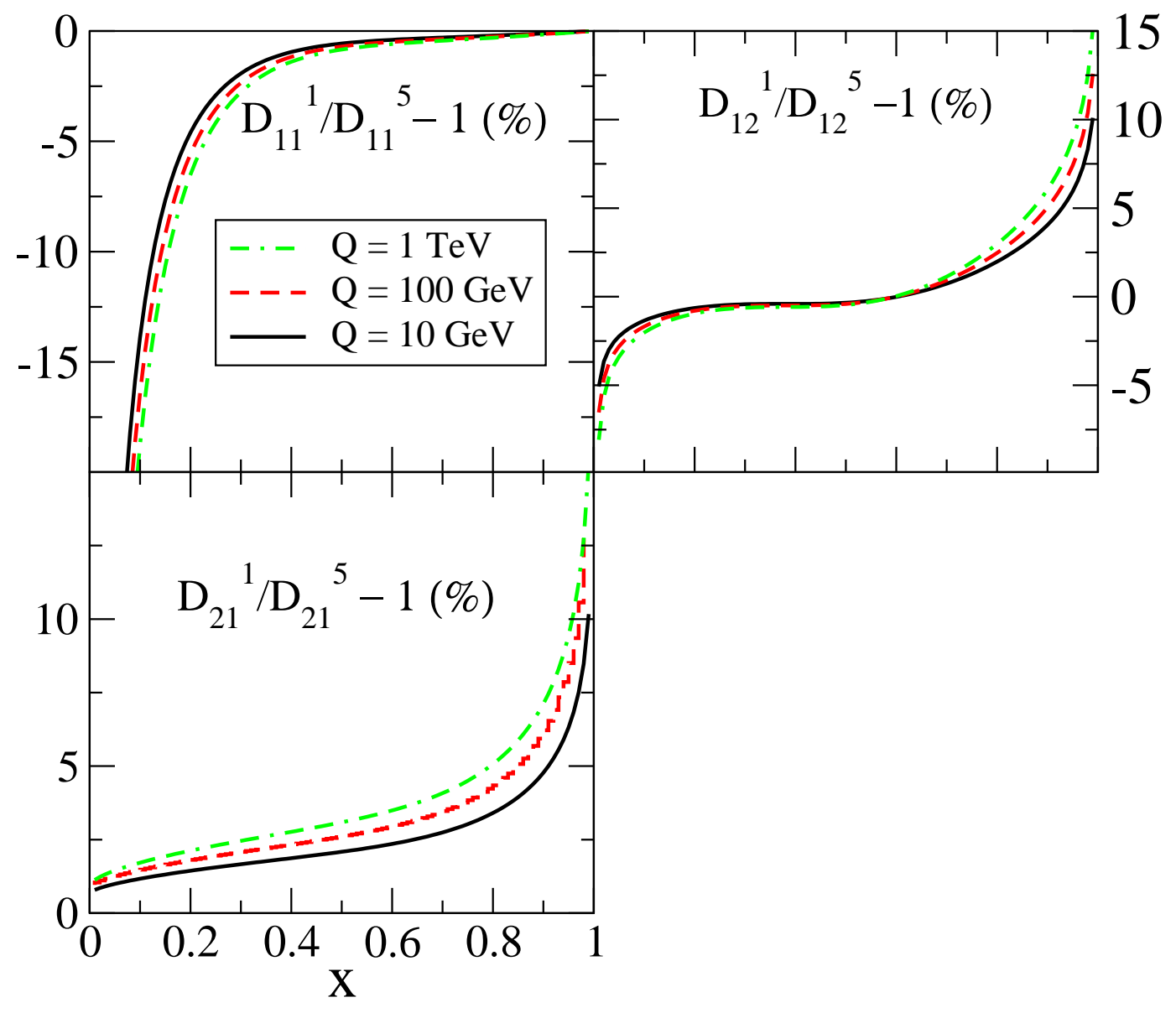

Figure 2: Relative contribution of the first order singlet radiators $D_{i j}^{1}$ in all terms to $O\left((\alpha L)^{5}\right)$. Here $D_{11}$ denotes the sum of the non-singlet contributions $D_{\mathrm{NS}}$, with soft exponentiation beyond $O\left((\alpha L)^{5}\right)$, and the pure singlet contribution $D_{11}^{\mathrm{PS}}$. 


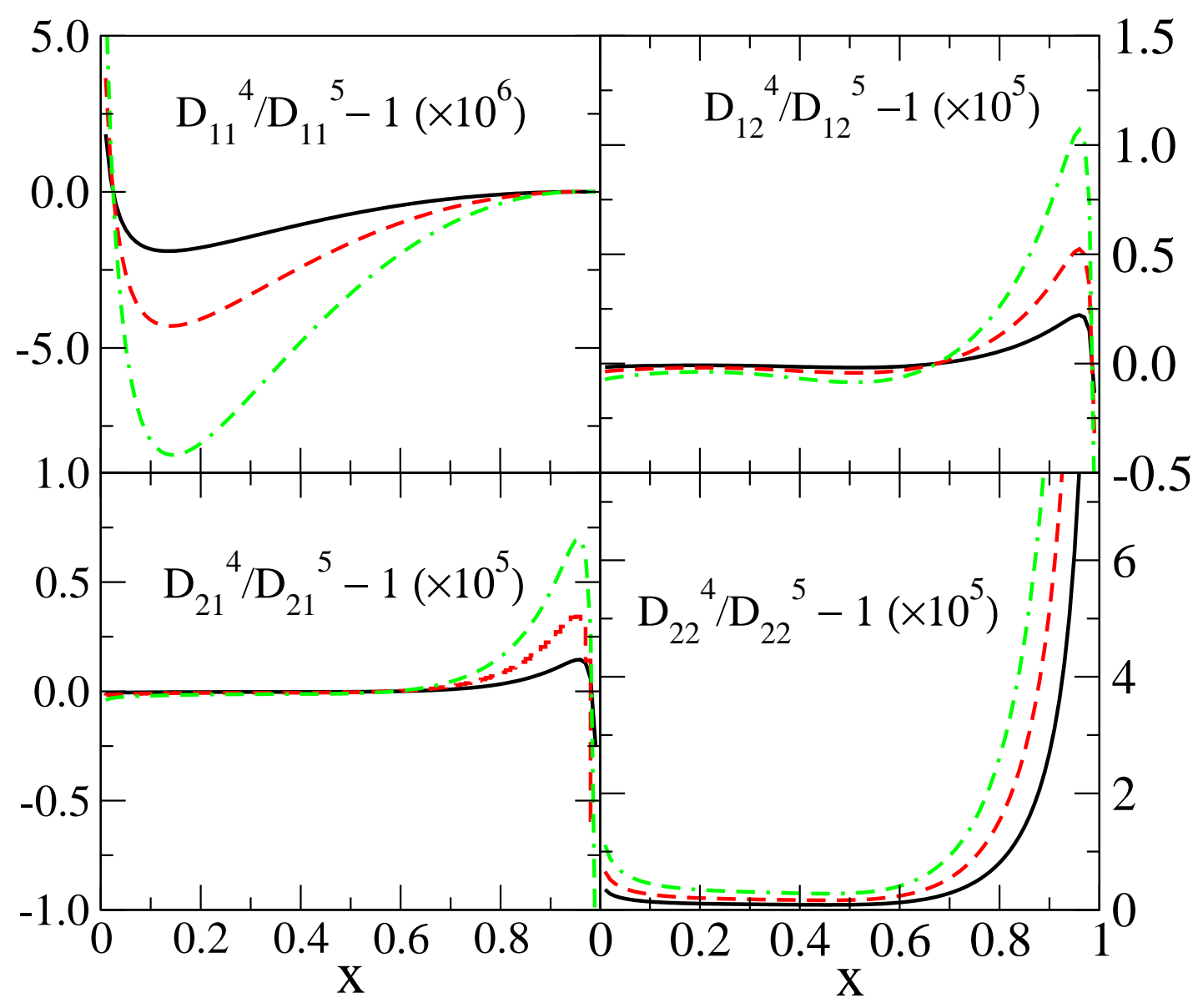

Figure 3: Relative contribution of the singlet radiators $D_{i j}$ up to the 4 th order in $\alpha L$ if compared to all terms to the 5 th order as a function of $x$ and $Q$, with $D_{11}=D_{11}^{\text {NS }+ \text { PS }}$. 


\section{Appendix A: Mellin Convolutions}

In this appendix we list the convolutions of functions up to weight 5 required in the present calculation in addition to those given in Ref. [15,25]. Some of the integrals require to use Mellin transforms and algebraic relations between the finite harmonic sums $[35,36]$. They were calculated recursively in explicit form and may be of general interest for other higher order calculations in QED and QCD.

$$
\begin{aligned}
& 1 \otimes \frac{1}{x}=\frac{1}{x}-1 \\
& x \otimes \frac{1}{x}=\frac{1}{2}\left(\frac{1}{x}-x\right) \\
& x^{2} \otimes \frac{1}{x}=\frac{1}{3}\left(\frac{1}{x}-x^{2}\right) \\
& \frac{1}{x} \otimes \frac{1}{x}=-\frac{1}{x} \ln (x) \\
& \left(\frac{1}{1-x}\right)_{+} \otimes \frac{1}{x}=\frac{1}{x} \ln (1-x) \\
& \left(\frac{\ln (1-x)}{1-x}\right)_{+} \otimes \frac{1}{x}=\frac{1}{2 x} \ln ^{2}(1-x) \\
& \ln (1-x) \otimes \frac{1}{x}=\left(\frac{1}{x}-1\right)[\ln (1-x)-1] \\
& x \ln (1-x) \otimes \frac{1}{x}=\frac{1}{2}\left(\frac{1}{x}-x\right) \ln (1-x)+\frac{x}{4}+\frac{1}{2}-\frac{3}{4 x} \\
& \ln (x) \otimes \frac{1}{x}=-\ln (x)+1-\frac{1}{x} \\
& x \ln (x) \otimes \frac{1}{x}=-\frac{x}{2} \ln (x)+\frac{1}{4}\left(x-\frac{1}{x}\right) \\
& \frac{\ln (x)}{1-x} \otimes \frac{1}{x}=-\frac{1}{x} \operatorname{Li}_{2}(1-x) \\
& \left(\frac{1}{1-x}\right)_{+} \otimes x^{2} \ln (1-x)=x^{2}\left[\ln ^{2}(1-x)-\ln (x) \ln (1-x)-\zeta_{2}\right] \\
& +\frac{1}{2}\left(1+2 x-3 x^{2}\right) \ln (1-x)+\frac{5}{4} x^{2}-\frac{x}{2}-\frac{3}{4} \\
& \left(\frac{1}{1-x}\right)_{+} \otimes x^{2} \ln (x)=x^{2}\left[-\frac{1}{2} \ln ^{2}(x)+\operatorname{Li}_{2}(1-x)+\ln (x) \ln (1-x)\right] \\
& -\frac{3}{2} x^{2} \ln (x)-\frac{1}{4}-x+\frac{5}{4} x^{2} \\
& 1 \otimes x^{2} \ln (1-x)=\frac{1}{2}\left(1-x^{2}\right) \ln (1-x)+\frac{x^{2}}{4}+\frac{x}{2}-\frac{3}{4} \\
& 1 \otimes x^{2} \ln (x)=-\frac{x^{2}}{2} \ln (x)-\frac{1}{4}\left(1-x^{2}\right) \\
& x \otimes x^{2} \ln (1-x)=x(1-x)[\ln (1-x)-1] \\
& x \otimes x^{2} \ln (x)=-x[1-x+x \ln (x)]
\end{aligned}
$$




$$
\begin{aligned}
& 1 \otimes x^{2}=\frac{1}{2}\left(1-x^{2}\right) \\
& x \otimes x^{2}=x(1-x) \\
& x^{2} \otimes x^{2}=-x^{2} \ln (x) \\
& x^{2} \otimes \ln (x)=\frac{1}{2} \ln (x)+\frac{1}{4}\left(1-x^{2}\right) \\
& x^{2} \otimes x \ln (x)=x[1-x+\ln (x)] \\
& \frac{1}{x} \ln (1-x) \otimes \frac{1}{x}=\frac{1}{x}\left[\operatorname{Li}_{2}(x)-\zeta_{2}\right] \\
& x^{2} \ln (1-x) \otimes \frac{1}{x}=\frac{1}{3}\left(\frac{1}{x}-x^{2}\right) \ln (1-x)-\frac{11}{18 x}+\frac{1}{3}+\frac{x}{6}+\frac{x^{2}}{9} \\
& x^{2} \ln (x) \otimes \frac{1}{x}=-\frac{x^{2}}{3} \ln (x)+\frac{1}{9}\left(x^{2}-\frac{1}{x}\right) \\
& \left(\frac{\ln ^{2}(1-x)}{1-x}\right)_{+} \otimes \frac{1}{x}=\frac{1}{3 x} \ln ^{3}(1-x) \\
& \ln ^{2}(1-x) \otimes \frac{1}{x}=\left(\frac{1}{x}-1\right)\left[\ln ^{2}(1-x)-2 \ln (1-x)+2\right] \\
& x \ln ^{2}(1-x) \otimes \frac{1}{x}=\frac{1}{2}\left(\frac{1}{x}-x\right) \ln ^{2}(1-x)+\left(\frac{x}{2}+1-\frac{3}{2 x}\right) \ln (1-x) \\
& +\frac{7}{4 x}-\frac{3}{2}-\frac{x}{4} \\
& x^{2} \ln ^{2}(1-x) \otimes \frac{1}{x}=\frac{1}{3}\left(\frac{1}{x}-x^{2}\right) \ln ^{2}(1-x)+\left(\frac{2}{9} x^{2}+\frac{x}{3}+\frac{2}{3}-\frac{11}{9 x}\right) \ln (1-x) \\
& +\frac{85}{54 x}-\frac{11}{9}-\frac{5}{18} x-\frac{2}{27} x^{2} \\
& \frac{\ln (x) \ln (1-x)}{1-x} \otimes \frac{1}{x}=\frac{1}{x}\left[\operatorname{Li}_{3}(1-x)-\ln (1-x) \operatorname{Li}_{2}(1-x)\right] \\
& \ln (x) \ln (1-x) \otimes \frac{1}{x}=-\frac{1}{x} \operatorname{Li}_{2}(1-x)-\ln (x) \ln (1-x)+\left(1-\frac{1}{x}\right) \ln (1-x) \\
& +\ln (x)-2\left(1-\frac{1}{x}\right) \\
& x \ln (x) \ln (1-x) \otimes \frac{1}{x}=-\frac{1}{2 x} \operatorname{Li}_{2}(1-x)-\frac{x}{2} \ln (x) \ln (1-x)+\frac{1}{4}\left(x-\frac{1}{x}\right) \ln (1-x) \\
& +\frac{1}{4}(2+x) \ln (x)+\frac{1}{x}-\frac{3}{4}-\frac{x}{4} \\
& x^{2} \ln (x) \ln (1-x) \otimes \frac{1}{x}=-\frac{1}{3 x} \operatorname{Li}_{2}(1-x)-\frac{x^{2}}{3} \ln (x) \ln (1-x)+\frac{1}{9}\left(x^{2}-\frac{1}{x}\right) \ln (1-x) \\
& +\frac{1}{18}\left(6+3 x+2 x^{2}\right) \ln (x)+\frac{71}{108 x}-\frac{4}{9}-\frac{5}{36} x-\frac{2}{27} x^{2} \\
& \frac{\ln ^{2}(x)}{1-x} \otimes \frac{1}{x}=\frac{2}{x} \mathrm{~S}_{1,2}(1-x) \\
& \ln ^{2}(x) \otimes \frac{1}{x}=\frac{2}{x}-2[1-\ln (x)]-\ln ^{2}(x)
\end{aligned}
$$




$$
\begin{aligned}
& x \ln ^{2}(x) \otimes \frac{1}{x}=\frac{1}{4}\left[\frac{1}{x}-x\right]+\frac{x}{2}\left[\ln (x)-\ln ^{2}(x)\right] \\
& x^{2} \ln ^{2}(x) \otimes \frac{1}{x}=\frac{2}{27}\left[\frac{1}{x}-x^{2}\right]+x^{2}\left[\frac{2}{9} \ln (x)-\frac{1}{3} \ln ^{2}(x)\right] \\
& \operatorname{Li}_{2}(1-x) \otimes \frac{1}{x}=\left(\frac{1}{x}-1\right)\left[\operatorname{Li}_{2}(1-x)-1\right]-\ln (x) \\
& x \operatorname{Li}_{2}(1-x) \otimes \frac{1}{x}=\frac{1}{2}\left(\frac{1}{x}-x\right) \operatorname{Li}_{2}(1-x)-\frac{1}{4}(2+x) \ln (x)-\frac{5}{8 x}+\frac{1}{2}+\frac{1}{8} x \\
& x^{2} \operatorname{Li}_{2}(1-x) \otimes \frac{1}{x}=\frac{1}{3}\left(\frac{1}{x}-x^{2}\right) \operatorname{Li}_{2}(1-x)-\left(\frac{1}{3}+\frac{x}{6}+\frac{x^{2}}{9}\right) \ln (x) \\
& -\frac{49}{108 x}+\frac{1}{3}+\frac{x}{12}+\frac{x^{2}}{27} \\
& \left(\frac{1}{1-x}\right)_{+} \otimes x^{2} \ln (x)=x^{2}\left[\zeta_{2}-\operatorname{Li}_{2}(x)-\frac{1}{2} \ln ^{2}(x)-\frac{3}{2} \ln (x)\right]-\frac{1}{4}-x+\frac{5}{4} x^{2} \\
& x^{2} \otimes\left(\frac{\ln ^{2}(1-x)}{1-x}\right)_{+}=\frac{x^{2}}{3} \ln ^{3}(1-x)+\left(\frac{1}{2}+x-\frac{3}{2} x^{2}\right) \ln ^{2}(1-x) \\
& -x(1-x) \ln (1-x)-x^{2} \ln (x) \\
& +2 x^{2}\left[\zeta_{3}-\mathrm{S}_{1,2}(x)\right]+3 x^{2}\left[\zeta_{2}-\mathrm{Li}_{2}(x)\right] \\
& x^{2} \otimes \ln ^{2}(1-x)=\frac{1}{2}\left(1-x^{2}\right) \ln ^{2}(1-x)-x(1-x) \ln (1-x) \\
& +x^{2}\left[\zeta_{2}-\mathrm{Li}_{2}(x)-\ln (x)\right] \\
& x^{2} \otimes x \ln ^{2}(1-x)=x(1-x) \ln ^{2}(1-x)-2 x^{2}\left[\operatorname{Li}_{2}(x)-\zeta_{2}\right] \\
& x^{2} \otimes \frac{\ln (x) \ln (1-x)}{1-x}=x^{2}\left[2 \zeta_{3}-\operatorname{Li}_{3}(x)-\mathrm{S}_{1,2}(x)\right]+\frac{3}{2} x^{2} \operatorname{Li}_{2}(1-x) \\
& +\frac{3}{4} x^{2} \ln ^{2}(x)+\frac{1}{4}\left(1+4 x-5 x^{2}\right) \ln (1-x) \\
& +\ln (x)\left[\frac{x^{2}}{2} \ln ^{2}(1-x)+x^{2} \operatorname{Li}_{2}(x)+\left(x+\frac{1}{2}\right) \ln (1-x)\right. \\
& \left.+\frac{5}{4} x^{2}-\frac{x}{2}\right]-\frac{3}{4} x(1-x) \\
& x^{2} \otimes \ln (x) \ln (1-x)=\frac{x^{2}}{2} \operatorname{Li}_{2}(1-x)+\frac{1}{4}\left(1-x^{2}\right) \ln (1-x)+\frac{x^{2}}{4} \ln ^{2}(x) \\
& +\frac{1}{2}\left[\ln (1-x)-x+\frac{x^{2}}{2}\right] \ln (x)-\frac{3}{4} x(1-x) \\
& x^{2} \otimes x \ln (x) \ln (1-x)=x(1-x)[1+\ln (x)] \ln (1-x)+x^{2}\left[\zeta_{2}-\operatorname{Li}_{2}(x)\right] \\
& +x^{2} \ln (x)\left(1+\frac{1}{2} \ln (x)\right) \\
& x^{2} \otimes \frac{\ln ^{2}(x)}{1-x}=2 x^{2} \mathrm{~S}_{1,2}(1-x)-\frac{x^{2}}{3} \ln ^{3}(x)+\left(\frac{1}{2}+x\right) \ln ^{2}(x) \\
& +\left(\frac{1}{2}+2 x\right) \ln (x)+\frac{1}{4}+2 x-\frac{9}{4} x^{2} \\
& x^{2} \otimes \ln ^{2}(x)=\frac{1}{4}\left(1-x^{2}\right)+\frac{1}{2}\left[\ln (x)+\ln ^{2}(x)\right]
\end{aligned}
$$




$$
\begin{aligned}
& x^{2} \otimes x \ln ^{2}(x)=2 x(1-x)+x\left[\ln ^{2}(x)+2 \ln (x)\right] \\
& x^{2} \otimes \operatorname{Li}_{2}(1-x)=\frac{1}{2}\left(1-x^{2}\right) \operatorname{Li}_{2}(1-x)+\frac{1}{2} x(1-x)+\frac{x}{2}\left[\ln (x)-\frac{x}{2} \ln ^{2}(x)\right](10 \\
& x^{2} \otimes x \operatorname{Li}_{2}(1-x)=x(1-x) \operatorname{Li}_{2}(1-x)-\frac{x^{2}}{2} \ln ^{2}(x) \\
& \left(\frac{\ln ^{3}(1-x)}{1-x}\right)_{+} \otimes \frac{1}{x}=\frac{1}{4 x} \ln ^{4}(1-x) \\
& \frac{\ln (x) \ln ^{2}(1-x)}{1-x} \otimes \frac{1}{x}=\frac{1}{x}\left\{\frac{1}{3} \ln (x) \ln ^{3}(1-x)+2\left[\mathrm{~S}_{1,3}(x)-\zeta_{4}\right]\right\} \\
& \frac{\ln ^{2}(x) \ln (1-x)}{1-x} \otimes \frac{1}{x}=\frac{1}{x}\left\{\frac{1}{2} \ln ^{2}(x) \ln ^{2}(1-x)+2\left[\mathrm{~S}_{2,2}(x)-\ln (x) \mathrm{S}_{1,2}(x)-\frac{\zeta_{4}}{4}\right]\right\}( \\
& \frac{\ln ^{3}(x)}{1-x} \otimes \frac{1}{x}=-\frac{6}{x} \mathrm{~S}_{1,3}(1-x) \\
& \frac{\mathrm{S}_{1,2}(1-x)}{1-x} \otimes \frac{1}{x}=\frac{1}{x} \mathrm{~S}_{2,2}(1-x) \\
& \frac{\mathrm{Li}_{3}(1-x)}{1-x} \otimes \frac{1}{x}=\frac{1}{x} \operatorname{Li}_{4}(1-x) \\
& \frac{\ln (x) \operatorname{Li}_{2}(1-x)}{1-x} \otimes \frac{1}{x}=-\frac{1}{2 x} \operatorname{Li}_{2}^{2}(1-x) \\
& \frac{\mathrm{Li}_{3}(x)-\zeta_{3}}{1-x} \otimes \frac{1}{x}=\frac{1}{x}\left\{\frac{1}{2}\left[\mathrm{Li}_{2}^{2}(x)-\zeta_{2}^{2}\right]+\ln (1-x)\left[\mathrm{Li}_{3}(x)-\zeta_{3}\right]\right\} \\
& \mathrm{S}_{1,2}(x) \otimes \frac{1}{x}=\frac{\zeta_{3}}{x}-\mathrm{S}_{1,2}(x)-\frac{1}{x}(1-x)\left[\frac{1}{2} \ln ^{2}(1-x)-\ln (1-x)+1\right] \\
& x \mathrm{~S}_{1,2}(x) \otimes \frac{1}{x}=\frac{1}{2 x}\left[\zeta_{3}-x^{2} \mathrm{~S}_{1,2}(x)\right]-\frac{1}{8 x}\left(1-x^{2}\right) \ln ^{2}(1-x) \\
& +\frac{1}{8 x}\left(3-2 x-x^{2}\right) \ln (1-x)-\frac{1}{x}\left(\frac{7}{16}-\frac{3}{8} x-\frac{1}{16} x^{2}\right) \\
& \operatorname{Li}_{3}(1-x) \otimes \frac{1}{x}=\left(\frac{1}{x}-1\right)\left[\operatorname{Li}_{3}(1-x)-\operatorname{Li}_{2}(1-x)+1\right]+\ln (x) \\
& x \operatorname{Li}_{3}(1-x) \otimes \frac{1}{x}=\frac{1}{2 x}\left(1-x^{2}\right) \operatorname{Li}_{3}(1-x)-\left(\frac{3}{4 x}-\frac{1}{2}-\frac{1}{4} x\right) \operatorname{Li}_{2}(1-x) \\
& +\left(\frac{3}{4}+\frac{x}{8}\right) \ln (x)+\frac{13}{16 x}-\frac{3}{4}-\frac{x}{16} \\
& \ln (1-x) \operatorname{Li}_{2}(1-x) \otimes \frac{1}{x}=-\frac{1}{x}(1-x)[1-\ln (1-x)] \operatorname{Li}_{2}(1-x) \\
& -\frac{1}{x}(1-x)[1-\ln (x)] \ln (1-x) \\
& +2 \ln (x)+\frac{1}{x}\left[\operatorname{Li}_{2}(x)-\zeta_{2}\right]+\frac{3}{x}(1-x) \\
& x \ln (1-x) \operatorname{Li}_{2}(1-x) \otimes \frac{1}{x}=\frac{1-x}{x}\left\{[\ln (1-x)-1]-\frac{1-x}{2}\left[\ln (1-x)-\frac{1}{2}\right]\right\} \operatorname{Li}_{2}(1-x) \\
& +\frac{1}{x}\left\{\left[\frac{3}{4}-\frac{x}{2}-\frac{x^{2}}{4}\right] \ln (1-x)+\frac{3}{2} x+\frac{x^{2}}{4}\right\} \ln (x)
\end{aligned}
$$




$$
\begin{aligned}
& -\frac{1}{8 x}\left[5-4 x-x^{2}\right] \ln (1-x)+\frac{3}{4 x}\left[\operatorname{Li}_{2}(x)-\zeta_{2}\right] \\
& +\frac{37}{16 x}-\frac{17}{8}-\frac{3}{16} x \\
& \ln (x) \operatorname{Li}_{2}(1-x) \otimes \frac{1}{x}=-\frac{1}{x}\left\{2 \mathrm{~S}_{1,2}(1-x)+x \ln ^{2}(x)\right. \\
& \left.+[1-x+x \ln (x)]\left[\operatorname{Li}_{2}(1-x)-3\right]\right\} \\
& x \ln (x) \operatorname{Li}_{2}(1-x) \otimes \frac{1}{x}=-\frac{1}{x} \mathrm{~S}_{1,2}(1-x)-\frac{1}{4 x}\left[1-x^{2}+2 x^{2} \ln (x)\right] \operatorname{Li}_{2}(1-x) \\
& -\frac{1}{4}(2+x) \ln ^{2}(x)+\frac{1}{8}(10+3 x) \ln (x) \\
& +\left(\frac{23}{16 x}-\frac{5}{4}-\frac{3}{16} x\right) \\
& \mathrm{S}_{1,2}(1-x) \otimes \frac{1}{x}=\left(\frac{1}{x}-1\right)\left[\mathrm{S}_{1,2}(1-x)-1\right]+\frac{1}{2} \ln ^{2}(x)-\ln (x) \\
& x \mathrm{~S}_{1,2}(1-x) \otimes \frac{1}{x}=\frac{1}{2}\left(\frac{1}{x}-x\right) \mathrm{S}_{1,2}(1-x)+\frac{1}{8}(2+x) \ln ^{2}(x) \\
& -\frac{1}{8}(4+x) \ln (x)-\frac{9}{16 x}+\frac{1}{2}+\frac{x}{16} \\
& \operatorname{Li}_{3}(x) \otimes \frac{1}{x}=-\left(\frac{1}{x}-1\right)[\ln (1-x)-1]-\frac{1}{x}\left(\zeta_{2}-\zeta_{3}\right)+\operatorname{Li}_{2}(x)-\operatorname{Li}_{3}(x)( \\
& x \operatorname{Li}_{3}(x) \otimes \frac{1}{x}=\frac{1}{x}\left(\frac{3}{16}-\frac{1}{4} \zeta_{2}+\frac{1}{2} \zeta_{3}\right)-\frac{1}{8}\left(1+\frac{x}{2}\right) \\
& -\frac{1}{8}\left(\frac{1}{x}-x\right) \ln (1-x)+x\left[\frac{1}{4} \mathrm{Li}_{2}(x)-\frac{1}{2} \mathrm{Li}_{3}(x)\right]
\end{aligned}
$$

$\ln (x) \mathrm{Li}_{2}(x) \otimes \frac{1}{x}=\frac{1}{x}\left\{x[1-\ln (x)] \mathrm{Li}_{2}(x)-\zeta_{2}-\mathrm{Li}_{2}(1-x)\right.$

$$
+3(1-x)-[2(1-x)+x \ln (x)] \ln (1-x)+x \ln (x)\}
$$

$$
\begin{aligned}
x \ln (x) \operatorname{Li}_{2}(x) \otimes \frac{1}{x}= & \frac{1}{4 x}\left\{x^{2}[1-2 \ln (x)] \operatorname{Li}_{2}(x)-\zeta_{2}\right\} \\
& -\frac{1}{4 x}\left[1-x^{2}+x^{2} \ln (x)\right] \ln (1-x) \\
& +\frac{11}{16 x}-\frac{1}{2}-\frac{3}{16} x+\frac{1}{8}(x+2) \ln (x)-\frac{1}{4 x} \operatorname{Li}_{2}(1-x) \\
\ln ^{3}(1-x) \otimes \frac{1}{x}= & \left(\frac{1}{x}-1\right)\left[\ln ^{3}(1-x)-3 \ln ^{2}(1-x)+6 \ln (1-x)-6\right]
\end{aligned}
$$$$
x \ln ^{3}(1-x) \otimes \frac{1}{x}=\frac{1}{2}\left(\frac{1}{x}-x\right) \ln ^{3}(1-x)+\left(-\frac{9}{4 x}+\frac{3}{2}+\frac{3}{4} x\right) \ln ^{2}(1-x)
$$$$
+\left(\frac{21}{4 x}-\frac{9}{2}-\frac{3}{4} x\right) \ln (1-x)-\frac{45}{8 x}+\frac{21}{4}+\frac{3}{8} x
$$

$\ln (x) \ln ^{2}(1-x) \otimes \frac{1}{x}=\frac{1}{x}(1-x)[\ln (x)-1] \ln ^{2}(1-x)$ 


$$
\begin{aligned}
& +\frac{4}{x}\left[1-x+\frac{x}{2} \ln (x)\right] \ln (1-x) \\
& -2 \ln (x)-\frac{2}{x}\left[\mathrm{~S}_{1,2}(x)-\zeta_{3}\right]+\frac{2}{x} \operatorname{Li}_{2}(1-x)-\frac{6}{x}(1-x) \\
& x \ln (x) \ln ^{2}(1-x) \otimes \frac{1}{x}=\frac{1}{x}\left\{\zeta_{3}-\mathrm{S}_{1,2}(x)-\frac{3}{2}\left[\operatorname{Li}_{2}(x)-\zeta_{2}\right]\right\} \\
& -\frac{1}{4 x}\left(1-x^{2}\right)[1-2 \ln (x)] \ln ^{2}(1-x) \\
& +\left\{\frac{2}{x}-\frac{3}{2}-\frac{x}{2}+\frac{1}{x}\left[-\frac{3}{2}+x+\frac{x^{2}}{2}\right] \ln (x)\right\} \ln (1-x) \\
& -\frac{1}{4}(x+6) \ln (x)-\frac{31}{8 x}+\frac{7}{2}+\frac{3}{8} x \\
& \ln ^{2}(x) \ln (1-x) \otimes \frac{1}{x}=\frac{2}{x}\left[\mathrm{~S}_{1,2}(1-x)+\operatorname{Li}_{2}(1-x)+\ln (1-x)-3(1-x)\right] \\
& +\ln (x)[\ln (x)-4]-\left[\ln ^{2}(x)-2 \ln (x)+2\right] \ln (1-x) \\
& x \ln ^{2}(x) \ln (1-x) \otimes \frac{1}{x}=\frac{1}{x}\left[\mathrm{~S}_{1,2}(1-x)+\frac{1}{2} \mathrm{Li}_{2}(1-x)\right] \\
& +\frac{1}{4 x}\left(1-x^{2}\right) \ln (1-x)+\frac{1}{2}\left[1+\frac{x}{2}-x \ln (1-x)\right] \ln ^{2}(x) \\
& -\frac{1}{2}[3+x-x \ln (1-x)] \ln (x)-\frac{17}{8 x}+\frac{7}{4}+\frac{3}{8} x \\
& \ln ^{3}(x) \otimes \frac{1}{x}=-6\left(\frac{1}{x}-1\right)-\ln ^{3}(x)+3 \ln ^{2}(x)-6 \ln (x) \\
& x \ln ^{3}(x) \otimes \frac{1}{x}=-\frac{3}{8}\left(\frac{1}{x}-x\right)-\frac{x}{2}\left[\ln ^{3}(x)-\frac{3}{2} \ln ^{2}(x)+\frac{3}{2} \ln (x)\right] \\
& \frac{1}{x} \operatorname{Li}_{2}(1-x) \otimes \frac{1}{x}=-\frac{1}{x}\left[2 \mathrm{~S}_{1,2}(1-x)+\ln (x) \operatorname{Li}_{2}(1-x)\right] \\
& \frac{1}{x} \operatorname{Li}_{2}(1-x) \otimes 1=\left(\frac{1}{x}-1\right) \operatorname{Li}_{2}(1-x)-\frac{1}{2} \ln ^{2}(x) \\
& \frac{1}{x} \operatorname{Li}_{2}(1-x) \otimes x=\frac{1}{2}(1-x)+\frac{1}{2}\left(\frac{1}{x}-x\right) \operatorname{Li}_{2}(1-x)+\frac{1}{2} \ln (x)\left[1-\frac{1}{2} x \ln (x)\right] \\
& \frac{1}{x} \operatorname{Li}_{2}(1-x) \otimes x^{2}=\frac{1}{3}\left(\frac{1}{x}-x^{2}\right) \operatorname{Li}_{2}(1-x) \\
& +\frac{1}{6} \ln (x)\left[1+2 x-x^{2} \ln (x)\right]+\frac{1}{12}\left[1+4 x-5 x^{2}\right] \\
& 1 \otimes x^{2} \ln ^{2}(1-x)=\frac{1}{2}\left(1-x^{2}\right) \ln ^{2}(1-x)+\frac{1}{2}\left(-3+2 x+x^{2}\right) \ln (1-x) \\
& +\frac{7}{4}-\frac{3}{2} x-\frac{x^{2}}{4} \\
& 1 \otimes x^{2} \ln (x) \ln (1-x)=\frac{1}{2}\left[\operatorname{Li}_{2}(x)-\zeta_{2}\right]+\frac{1}{2}\left(1-x^{2}\right) \ln (x) \ln (1-x) \\
& -\frac{1}{4}\left(1-x^{2}\right) \ln (1-x)+\frac{x}{4}(2+x) \ln (x)+1-\frac{3}{4} x-\frac{x^{2}}{4} \\
& 1 \otimes x^{2} \ln ^{2}(x)=\frac{x^{2}}{2}\left[\ln (x)-\ln ^{2}(x)\right]+\frac{1}{4}\left(1-x^{2}\right)
\end{aligned}
$$




$$
\begin{aligned}
& 1 \otimes \frac{1}{x} \ln (1-x)=\left(\frac{1}{x}-1\right) \ln (1-x)+\ln (x) \\
& 1 \otimes x^{2} \operatorname{Li}_{2}(1-x)=\frac{1}{2}\left(1-x^{2}\right) \operatorname{Li}_{2}(1-x)-\frac{x}{4}(2+x) \ln (x)-\frac{5}{8}+\frac{x}{2}+\frac{x^{2}}{8} \\
& x \otimes x^{2} \ln ^{2}(1-x)=x(1-x)\left[\ln ^{2}(1-x)-2 \ln (1-x)+2\right] \\
& x \otimes x^{2} \ln (x) \ln (1-x)=-x \operatorname{Li}_{2}(1-x)+x(1-x)[2-\ln (1-x)] \\
& +x^{2} \ln (x)[1-\ln (1-x)] \\
& x \otimes x^{2} \ln ^{2}(x)=-x^{2} \ln ^{2}(x)+2 x[x \ln (x)-x+1] \\
& x \otimes \frac{1}{x} \ln (1-x)=\frac{1}{2}\left(\frac{1}{x}-x\right) \ln (1-x)+\frac{x}{2} \ln (x)-\frac{1}{2}(1-x) \\
& x^{2} \otimes \frac{1}{(1-x)_{+}}=x^{2}[\ln (1-x)-\ln (x)]+\frac{1}{2}+x-\frac{3}{2} x^{2} \\
& x^{2} \otimes \ln (1-x)=\frac{1}{2}\left(1-x^{2}\right) \ln (1-x)+\frac{x}{2}[x \ln (x)+x-1] \\
& x^{2} \otimes x \ln (1-x)=x(1-x) \ln (1-x)+x^{2} \ln (x) \\
& x^{2} \otimes\left(\frac{\ln (1-x)}{1-x}\right)_{+}=-x^{2} \operatorname{Li}_{2}(1-x)+\frac{x^{2}}{2} \ln ^{2}(1-x)-x^{2} \ln (x) \ln (1-x) \\
& +\frac{3}{2} x^{2} \ln (x)+\left(\frac{1}{2}+x-\frac{3}{2} x^{2}\right) \ln (1-x)-\frac{1}{2} x(1-x) \\
& x^{2} \otimes \frac{\ln (x)}{1-x}=-x^{2} \operatorname{Li}_{2}(1-x)-\frac{x^{2}}{2} \ln ^{2}(x)+\left(x+\frac{1}{2}\right) \ln (x) \\
& +\frac{1}{4}\left(1+4 x-5 x^{2}\right) \\
& x^{2} \otimes \frac{1}{x} \ln (1-x)=\frac{1}{3}\left(\frac{1}{x}-x^{2}\right) \ln (1-x)+\frac{x^{2}}{3} \ln (x)-\frac{1}{6}-\frac{x}{3}+\frac{x^{2}}{2} \\
& x^{2} \otimes x^{2} \ln (1-x)=x^{2}\left[\operatorname{Li}_{2}(x)-\zeta_{2}\right] \\
& x^{2} \otimes x^{2} \ln (x)=-\frac{x^{2}}{2} \ln ^{2}(x) \\
& x \otimes x^{2} \operatorname{Li}_{2}(1-x)=x(1-x)\left[\operatorname{Li}_{2}(1-x)-1\right]-x^{2} \ln (x) \\
& x^{2} \otimes\left(\frac{\ln ^{3}(1-x)}{1-x}\right)_{+}=3 x^{2}\left\{2\left[\mathrm{~S}_{1,3}(x)-\zeta_{4}\right]+3\left[\mathrm{~S}_{1,2}(x)-\zeta_{3}\right]+\left[\operatorname{Li}_{2}(x)-\zeta_{2}\right]\right\} \\
& +\frac{x^{2}}{4} \ln ^{4}(1-x)+\frac{1}{2}\left(1+2 x-3 x^{2}\right) \ln ^{3}(1-x) \\
& -\frac{3}{2} x(1-x) \ln ^{2}(1-x) \\
& x^{2} \otimes \frac{\ln (x) \ln ^{2}(1-x)}{1-x}=2 x^{2}\left[\mathrm{~S}_{2,2}(x)+\mathrm{S}_{1,3}(x)-\ln (x) \mathrm{S}_{1,2}(x)-\frac{5}{4} \zeta_{4}\right] \\
& +3 x^{2}\left[\mathrm{~S}_{1,2}(x)-\mathrm{S}_{1,2}(1-x)-\zeta_{3}\right] \\
& -2 x^{2}\left[\operatorname{Li}_{2}(x)-\zeta_{2}\right]-\frac{x^{2}}{2} \operatorname{Li}_{2}(1-x) \\
& +\frac{x^{2}}{3} \ln (x) \ln ^{3}(1-x)-\frac{1}{2} x^{2}\left[\ln ^{2}(x)+3 \ln (x)\right]
\end{aligned}
$$




$$
\begin{aligned}
& +\left[\frac{1}{4}+x-\frac{5}{4} x^{2}+\left(\frac{1}{2}+x-\frac{3}{2} x^{2}\right) \ln (x)\right] \ln ^{2}(1-x) \\
& +\left[-\frac{3}{2} x(1-x)+\left(\frac{x^{2}}{2}-x\right) \ln (x)+\frac{3}{2} x^{2} \ln ^{2}(x)\right] \ln (1-x) \\
& x^{2} \otimes \frac{\ln ^{2}(x) \ln (1-x)}{1-x}=2 x^{2}\left[\left\{\ln (1-x)-\frac{3}{2}\right\} \mathrm{S}_{1,2}(1-x)-\mathrm{S}_{2,2}(1-x)-\mathrm{S}_{1,3}(1-x)\right] \\
& +\frac{5}{2} x^{2} \operatorname{Li}_{2}(1-x)+x^{2}\left(\frac{1}{2}-\frac{1}{3} \ln (1-x)\right) \ln ^{3}(x) \\
& +\left[-\frac{x}{2}+\frac{5}{4} x^{2}+\left(\frac{1}{2}+x\right) \ln (1-x)\right] \ln ^{2}(x) \\
& +\left[-\frac{3}{2} x+\frac{9}{4} x^{2}+\left(\frac{1}{2}+2 x\right) \ln (1-x)\right] \ln (x) \\
& +\left(\frac{1}{4}+2 x-\frac{9}{4} x^{2}\right) \ln (1-x)-\frac{7}{4} x(1-x) \\
& x^{2} \otimes \frac{\ln ^{3}(x)}{1-x}=-6 x^{2} \mathrm{~S}_{1,3}(1-x)-\frac{x^{2}}{4} \ln ^{4}(x)+\left(x+\frac{1}{2}\right) \ln ^{3}(x) \\
& +3\left(x+\frac{1}{4}\right) \ln ^{2}(x)+6\left(x+\frac{1}{8}\right) \ln (x)+\frac{3}{8}+6 x-\frac{51}{8} x^{2} \\
& x^{2} \otimes \frac{\ln (x) \mathrm{Li}_{2}(1-x)}{1-x}=3 x^{2}\left[\mathrm{~S}_{1,3}(1-x)+\mathrm{S}_{1,2}(1-x)-\frac{1}{6} \mathrm{Li}_{2}^{2}(1-x)\right] \\
& +\frac{1}{2}\left[-x^{2} \ln ^{2}(x)+(1+2 x) \ln (x)+\frac{1}{2}+2 x-\frac{5}{2} x^{2}\right] \operatorname{Li}_{2}(1-x) \\
& -\frac{x^{2}}{2} \ln ^{3}(x)+\frac{x}{2}\left(1-\frac{5}{4} x\right) \ln ^{2}(x)+\frac{5}{4} x \ln (x)+\frac{5}{4} x(1-x) \\
& x^{2} \otimes \operatorname{Li}_{3}(1-x)=\frac{1}{2}\left(1-x^{2}\right) \operatorname{Li}_{3}(1-x)-\frac{1}{2}\left[x(1-x)-x^{2} \ln (x)\right] \operatorname{Li}_{2}(1-x) \\
& +\frac{x^{2}}{4} \ln ^{2}(x)+x^{2} \mathrm{~S}_{1,2}(1-x) \\
& x^{2} \otimes x \operatorname{Li}_{3}(1-x)=x(1-x) \operatorname{Li}_{3}(1-x)+x^{2} \ln (x) \operatorname{Li}_{2}(1-x)+2 x^{2} \mathrm{~S}_{1,2}(1-x) \\
& x^{2} \otimes \ln (1-x) \operatorname{Li}_{2}(1-x)=\frac{3}{2} x^{2} \mathrm{~S}_{1,2}(1-x) \\
& +\frac{1}{2}\left[\left(1-x^{2}\right) \ln (1-x)+x^{2} \ln (x)-x+2 x^{2}\right] \operatorname{Li}_{2}(1-x) \\
& +\frac{x}{2}\left[-\frac{x}{2} \ln ^{2}(x)+\ln (x)+1-x\right] \ln (1-x) \\
& +\frac{x^{2}}{2}\left[\ln ^{2}(x)+\ln (x)\right] \\
& x^{2} \otimes x \ln (1-x) \operatorname{Li}_{2}(1-x)=x^{2}\left[2 \mathrm{~S}_{1,2}(1-x)+\ln (x) \operatorname{Li}_{2}(x)-\operatorname{Li}_{3}(x)+\zeta_{3}\right] \\
& +x[x \ln (x)+(1-x) \ln (1-x)] \operatorname{Li}_{2}(1-x) \\
& x^{2} \otimes \ln (x) \operatorname{Li}_{2}(1-x)=x^{2} \mathrm{~S}_{1,2}(1-x)+\frac{1}{2}\left[\ln (x)+\frac{1}{2}\left(1-x^{2}\right)\right] \operatorname{Li}_{2}(1-x) \\
& -\frac{x^{2}}{6} \ln ^{3}(x)+\frac{x}{8}(4-x) \ln ^{2}(x)+\frac{5}{4} x[1-x+\ln (x)]
\end{aligned}
$$




$$
\begin{aligned}
x^{2} \otimes x \ln (x) \mathrm{Li}_{2}(1-x)= & 2 x^{2} \mathrm{~S}_{1,2}(1-x)+x[1-x+\ln (x)] \mathrm{Li}_{2}(1-x) \\
& -x^{2}\left[\frac{1}{3} \ln ^{3}(x)+\frac{1}{2} \ln ^{2}(x)\right] \\
x^{2} \otimes \mathrm{S}_{1,2}(1-x)= & \frac{1}{2}\left(1-x^{2}\right) \mathrm{S}_{1,2}(1-x)+\frac{x^{2}}{12} \ln ^{3}(x)-\frac{x}{4} \ln ^{2}(x)-\frac{x}{2} \ln (x) \\
& -\frac{x}{2}(1-x) \\
x^{2} \otimes x \mathrm{~S}_{1,2}(1-x)= & x(1-x) \mathrm{S}_{1,2}(1-x)+\frac{x^{2}}{6} \ln ^{3}(x) \\
x^{2} \otimes \ln ^{3}(1-x)= & \frac{1}{2}\left(1-x^{2}\right) \ln ^{3}(1-x)-\frac{3}{2} x(1-x) \ln ^{2}(1-x) \\
& +3 x^{2}\left[\mathrm{~S}_{1,2}(x)-\zeta_{3}+\mathrm{Li}_{2}(x)-\zeta_{2}\right] \\
x^{2} \otimes x \ln ^{3}(1-x)= & x(1-x) \ln ^{3}(1-x)+6 x^{2}\left[\mathrm{~S}_{1,2}(x)-\zeta_{3}\right] \\
x^{2} \otimes \ln (x) \ln ^{2}(1-x)= & x^{2}\left[\mathrm{~S}_{1,2}(x)-\mathrm{S}_{1,2}(1-x)-\zeta_{3}\right]-\frac{x^{2}}{2} \operatorname{Li}_{2}(1-x) \\
& +\frac{1}{2}\left[\frac{1}{2}+\ln (x)\right]\left(1-x^{2}\right) \ln ^{2}(1-x) \\
& +\left[-\frac{3}{2} x(1-x)-x\left(1-\frac{x}{2}\right) \ln (x)+\frac{x^{2}}{2} \ln ^{2}(x)\right] \ln (1-x) \\
& -\frac{x^{2}}{2}\left[3 \ln (x)+\ln ^{2}(x)\right] \\
& (1-x)[1+\ln (x)] \ln ^{2}(1-x)+2 x^{2} \ln ^{2}(x) \ln (1-x)
\end{aligned}
$$

$x^{2} \otimes x \ln (x) \ln ^{2}(1-x)=x(1-x)[1+\ln (x)] \ln ^{2}(1-x)+2 x^{2} \ln (x) \ln (1-x)$ $+2 x^{2}\left[\mathrm{~S}_{1,2}(x)+\operatorname{Li}_{3}(x)+\operatorname{Li}_{2}(1-x)-\ln (x) \operatorname{Li}_{2}(x)-2 \zeta_{3}\right]$

$$
x^{2} \otimes \ln ^{2}(x) \ln (1-x)=-x^{2} \mathrm{~S}_{1,2}(1-x)+\frac{x^{2}}{2} \operatorname{Li}_{2}(1-x)
$$$$
+\left\{\frac{1}{4}\left(1-x^{2}\right)+\frac{1}{2} \ln (x)[1+\ln (x)]\right\} \ln (1-x)
$$$$
+\frac{x^{2}}{6} \ln ^{3}(x)-\frac{x}{2}\left(1-\frac{x}{2}\right) \ln ^{2}(x)-\frac{x}{2}\left(3-\frac{x}{2}\right) \ln (x)
$$$$
-\frac{7}{4} x(1-x)
$$

$x^{2} \otimes x \ln ^{2}(x) \ln (1-x)=x\left[2(1-x)+\ln ^{2}(x)+2 \ln (x)\right] \ln (1-x)$

$$
\begin{aligned}
& +2 x^{2}\left[\frac{1}{6} \ln ^{3}(x)+\frac{1}{2} \ln ^{2}(x)+\ln (x)\right] \\
& +2 x^{2}\left[\operatorname{Li}_{2}(1-x)-\mathrm{S}_{1,2}(1-x)\right] \\
x^{2} \otimes \ln ^{3}(x)= & \frac{1}{2} \ln ^{3}(x)+\frac{3}{4} \ln ^{2}(x)+\frac{3}{4} \ln (x)+\frac{3}{8}\left(1-x^{2}\right) \\
x^{2} \otimes x \ln ^{3}(x)= & x\left[\ln ^{3}(x)+3 \ln ^{2}(x)+6 \ln (x)\right]+6 x(1-x) \\
x^{2} \mathrm{~S}_{1,2}(1-x) \otimes \frac{1}{x}= & \frac{1}{3}\left(\frac{1}{x}-x^{2}\right) \mathrm{S}_{1,2}(1-x)+\frac{1}{6}\left[1+\frac{x}{2}+\frac{x^{2}}{3}\right] \ln ^{2}(x) \\
& -\frac{1}{3}\left[1+\frac{x}{4}+\frac{x^{2}}{9}\right] \ln (x)-\frac{251}{648 x}+\frac{1}{3}+\frac{x}{24}+\frac{x^{2}}{81} \\
x^{2} \operatorname{Li}_{3}(1-x) \otimes \frac{1}{x}= & \frac{1}{3}\left(\frac{1}{x}-x^{2}\right) \operatorname{Li}_{3}(1-x)+\left[-\frac{11}{18 x}+\frac{1}{3}+\frac{x}{6}+\frac{x^{2}}{9}\right] \operatorname{Li}_{2}(1-x)
\end{aligned}
$$




$$
\begin{aligned}
& +\left[\frac{11}{18}+\frac{5}{36} x+\frac{x^{2}}{27}\right] \ln (x)+\frac{449}{648 x}-\frac{11}{18}-\frac{5}{72} x-\frac{x^{2}}{81} \\
& \frac{1}{x} \ln ^{2}(1-x) \otimes \frac{1}{x}=\frac{2}{x}\left[\zeta_{3}-\mathrm{S}_{1,2}(x)\right] \\
& \frac{1}{x} \ln (x) \otimes \frac{1}{x}=-\frac{1}{2 x} \ln ^{2}(x) \\
& 1 \otimes x^{2} \mathrm{~S}_{1,2}(1-x)=\frac{1}{2}\left(1-x^{2}\right) \mathrm{S}_{1,2}(1-x)+\frac{x}{4}\left(1+\frac{x}{2}\right) \ln ^{2}(x) \\
& -\frac{x}{2}\left(1+\frac{x}{4}\right) \ln (x)-\frac{9}{16}+\frac{x}{2}+\frac{x^{2}}{16} \\
& x \otimes x^{2} \mathrm{~S}_{1,2}(1-x)=x(1-x)\left[\mathrm{S}_{1,2}(1-x)-1\right]+x^{2} \ln (x)\left[\frac{1}{2} \ln (x)-1\right] \\
& 1 \otimes x^{2} \operatorname{Li}_{3}(1-x)=\frac{1}{2}\left(1-x^{2}\right) \operatorname{Li}_{3}(1-x)-\frac{1}{4}\left(3-2 x-x^{2}\right) \operatorname{Li}_{2}(1-x) \\
& +\frac{x}{4}\left(3+\frac{x}{2}\right) \ln (x)+\frac{13}{16}-\frac{3}{4} x-\frac{x^{2}}{16} \\
& x \otimes x^{2} \operatorname{Li}_{3}(1-x)=x(1-x)\left[\operatorname{Li}_{3}(1-x)-\operatorname{Li}_{2}(1-x)+1\right]+x^{2} \ln (x) \\
& x^{2} \otimes x^{2} \operatorname{Li}_{3}(1-x)=-x^{2} \ln (x) \operatorname{Li}_{3}(1-x)-\frac{x^{2}}{2} \operatorname{Li}_{2}^{2}(1-x) \\
& 1 \otimes \frac{1}{x} \ln ^{2}(1-x)=\left(\frac{1}{x}-1\right) \ln ^{2}(1-x)-2\left[\operatorname{Li}_{2}(x)-\zeta_{2}\right] \\
& x \otimes \frac{1}{x} \ln ^{2}(1-x)=\frac{1}{2}\left(\frac{1}{x}-x\right) \ln ^{2}(1-x)-(1-x) \ln (1-x)-x \ln (x) \\
& -x\left[\mathrm{Li}_{2}(x)-\zeta_{2}\right] \\
& x^{2} \otimes \frac{1}{x} \ln ^{2}(1-x)=\frac{1}{3}\left(\frac{1}{x}-x^{2}\right) \ln ^{2}(1-x)-\frac{1}{3}\left(1+2 x-3 x^{2}\right) \ln (1-x) \\
& -x^{2} \ln (x)-\frac{2}{3} x^{2}\left[\operatorname{Li}_{2}(x)-\zeta_{2}\right]+\frac{1}{3} x(1-x) \\
& 1 \otimes \frac{1}{x} \ln (x)=\frac{1}{x}[1-x+\ln (x)] \\
& x \otimes \frac{1}{x} \ln (x)=\frac{1}{2 x}\left[\frac{1}{2}\left(1-x^{2}\right)+\ln (x)\right] \\
& x^{2} \otimes \frac{1}{x} \ln (x)=\frac{1}{3 x}\left[\frac{1}{3}\left(1-x^{3}\right)+\ln (x)\right] \\
& x^{2} \ln (1-x) \operatorname{Li}_{2}(1-x) \otimes \frac{1}{x}=\left\{-\frac{11}{18 x}+\frac{1}{3}+\frac{x}{6}+\frac{x^{2}}{9}+\frac{1}{3}\left(\frac{1}{x}-x^{2}\right) \ln (1-x)\right\} \operatorname{Li}_{2}(1-x) \\
& +\frac{11}{18 x}\left[\operatorname{Li}_{2}(x)-\zeta_{2}\right]+\left(\frac{11}{18 x}-\frac{1}{3}-\frac{x}{6}-\frac{x^{2}}{9}\right) \ln (x) \ln (1-x) \\
& +\left(-\frac{49}{108 x}+\frac{1}{3}+\frac{x}{12}+\frac{x^{2}}{27}\right) \ln (1-x) \\
& +\left(\frac{11}{9}+\frac{5}{18} x+\frac{2}{27} x^{2}\right) \ln (x)+\frac{413}{216 x}-\frac{181}{108}-\frac{43}{216} x-\frac{x^{2}}{27} \\
& x^{2} \ln ^{3}(1-x) \otimes \frac{1}{x}=\frac{1}{3}\left(\frac{1}{x}-x^{2}\right) \ln ^{3}(1-x)+\left(-\frac{11}{6 x}+1+\frac{x}{2}+\frac{x^{2}}{3}\right) \ln ^{2}(1-x)
\end{aligned}
$$




$$
\begin{aligned}
& +\left(\frac{85}{18 x}-\frac{11}{3}-\frac{5}{6} x-\frac{2}{9} x^{2}\right) \ln (1-x) \\
& -\frac{575}{108 x}+\frac{85}{18}+\frac{19}{36} x+\frac{2}{27} x^{2} \\
& x^{2} \ln (x) \ln ^{2}(1-x) \otimes \frac{1}{x}=\frac{2}{3 x}\left[\zeta_{3}-\mathrm{S}_{1,2}(x)\right]-\frac{11}{9 x}\left[\operatorname{Li}_{2}(x)-\zeta_{2}\right] \\
& +\frac{1}{3}\left(\frac{1}{x}-x^{2}\right) \ln (x) \ln ^{2}(1-x)-\frac{1}{9}\left(\frac{1}{x}-x^{2}\right) \ln ^{2}(1-x) \\
& +\left(-\frac{11}{9 x}+\frac{2}{3}+\frac{x}{3}+\frac{2}{9} x^{2}\right) \ln (x) \ln (1-x) \\
& +\left(\frac{71}{54 x}-\frac{8}{9}-\frac{5}{18} x-\frac{4}{27} x^{2}\right) \ln (1-x) \\
& -\left(\frac{11}{9}+\frac{5}{18} x+\frac{2}{27} x^{2}\right) \ln (x)-\frac{80}{27 x}+\frac{137}{54}+\frac{19}{54} x+\frac{2}{27} x^{2} \\
& x^{2} \ln ^{2}(x) \ln (1-x) \otimes \frac{1}{x}=\frac{2}{3 x} \mathrm{~S}_{1,2}(1-x)+\frac{2}{9 x} \operatorname{Li}_{2}(1-x) \\
& +\left(\frac{1}{3}+\frac{x}{6}+\frac{x^{2}}{9}-\frac{x^{2}}{3} \ln (1-x)\right) \ln ^{2}(x) \\
& +\frac{2}{9} x^{2} \ln (x) \ln (1-x)-\left(\frac{8}{9}+\frac{5}{18} x+\frac{4}{27} x^{2}\right) \ln (x) \\
& +\frac{2}{27}\left(\frac{1}{x}-x^{2}\right) \ln (1-x)-\frac{131}{108 x}+\frac{26}{27}+\frac{19}{108} x+\frac{2}{27} x^{2} \\
& x^{2} \ln ^{3}(x) \otimes \frac{1}{x}=-\frac{2}{27}\left(\frac{1}{x}-x^{2}\right)-\frac{x^{2}}{3}\left(\frac{2}{3} \ln (x)-\ln ^{2}(x)+\ln ^{3}(x)\right) \\
& 1 \otimes x^{2} \ln (1-x) \operatorname{Li}_{2}(1-x)=\frac{1-x}{2}\left\{(1+x) \ln (1-x)-\frac{3}{2}-\frac{x}{2}\right\} \operatorname{Li}_{2}(1-x) \\
& +\frac{3}{4}\left[\operatorname{Li}_{2}(x)-\zeta_{2}\right]+\left(\frac{3}{4}-\frac{x}{2}-\frac{x^{2}}{4}\right) \ln (x) \ln (1-x) \\
& +\left(\frac{3}{2}+\frac{x}{4}\right) x \ln (x)-\frac{1}{8}\left(5-4 x-x^{2}\right) \ln (1-x) \\
& +\frac{37}{16}-\frac{17}{8} x-\frac{3}{16} x^{2} \\
& 1 \otimes x^{2} \ln ^{3}(1-x)=\frac{1}{2}\left(1-x^{2}\right) \ln ^{3}(1-x)+\left(-\frac{9}{4}+\frac{3}{2} x+\frac{3}{4} x^{2}\right) \ln ^{2}(1-x) \\
& +\left(\frac{21}{4}-\frac{9}{2} x-\frac{3}{4} x^{2}\right) \ln (1-x)-\frac{45}{8}+\frac{21}{4} x+\frac{3}{8} x^{2} \\
& 1 \otimes x^{2} \ln (x) \ln ^{2}(1-x)=\zeta_{3}-\mathrm{S}_{1,2}(x)-\frac{3}{2}\left[\operatorname{Li}_{2}(x)-\zeta_{2}\right] \\
& -\frac{1}{4}\left(1-x^{2}\right)[1-2 \ln (x)] \ln ^{2}(1-x) \\
& +\left(-\frac{3}{2}+x+\frac{x^{2}}{2}\right) \ln (x) \ln (1-x)+\left(2-\frac{3}{2} x-\frac{x^{2}}{2}\right) \ln (1-x) \\
& -\frac{x}{4}(x+6) \ln (x)-\frac{31}{8}+\frac{7}{2} x+\frac{3}{8} x^{2}
\end{aligned}
$$




$$
\begin{aligned}
& 1 \otimes x^{2} \ln ^{2}(x) \ln (1-x)=\mathrm{S}_{1,2}(1-x)+\frac{1}{2} \mathrm{Li}_{2}(1-x) \\
& +\frac{x}{2}\left[1+\frac{x}{2}-x \ln (1-x)\right] \ln ^{2}(x) \\
& -\frac{x}{2}[3+x-x \ln (1-x)] \ln (x) \\
& +\frac{1}{4}\left(1-x^{2}\right) \ln (1-x)-\frac{17}{8}+\frac{7}{4} x+\frac{3}{8} x^{2} \\
& 1 \otimes x^{2} \ln ^{3}(x)=-\frac{x^{2}}{2}\left\{\ln ^{3}(x)-\frac{3}{2} \ln ^{2}(x)+\frac{3}{2} \ln (x)\right\}-\frac{3}{8}\left(1-x^{2}\right) \\
& x \otimes x^{2} \ln (1-x) \operatorname{Li}_{2}(1-x)=-x \operatorname{Li}_{2}(1-x)+x(1-x)[\ln (1-x)-1] \operatorname{Li}_{2}(1-x) \\
& +x(1-x)[3-\ln (1-x)]+x^{2} \ln (x)[2-\ln (1-x)] \\
& x \otimes x^{2} \ln ^{3}(1-x)=-x(1-x)\left[6-6 \ln (1-x)+3 \ln ^{2}(1-x)-\ln ^{3}(1-x)\right] \\
& x \otimes x^{2} \ln (x) \ln ^{2}(1-x)=2 x\left[\zeta_{3}-\mathrm{S}_{1,2}(x)\right]+2 x\left[\zeta_{2}-\mathrm{Li}_{2}(x)\right]-2 x \ln (x) \\
& -x(1-x)\left[6-4 \ln (1-x)+\ln ^{2}(1-x)\right] \\
& +x(1-x) \ln (x)\left[2-2 \ln (1-x)+\ln ^{2}(1-x)\right] \\
& x \otimes x^{2} \ln ^{2}(x) \ln (1-x)=2 x\left[\mathrm{~S}_{1,2}(1-x)+\operatorname{Li}_{2}(1-x)\right] \\
& +x^{2} \ln (x)\{-2+[2-\ln (x)][\ln (1-x)-1]\} \\
& +2 x(1-x)[\ln (1-x)-3] \\
& x \otimes x^{2} \ln ^{3}(x)=-x^{2} \ln ^{3}(x)+3 x^{2} \ln ^{2}(x)-6 x(x \ln (x)-x+1) \\
& x^{2} \otimes x^{2} \ln ^{2}(1-x)=2 x^{2}\left[\zeta(3)-\mathrm{S}_{1,2}(x)\right] \\
& x^{2} \otimes x^{2} \ln (x) \ln (1-x)=x^{2}\left[\zeta_{3}+\ln (x) \operatorname{Li}_{2}(x)-\operatorname{Li}_{3}(x)\right] \\
& x^{2} \otimes x^{2} \ln ^{2}(x)=-\frac{x^{2}}{3} \ln ^{3}(x) \\
& x^{2} \otimes x^{2} \operatorname{Li}_{2}(1-x)=-x^{2}\left[2 \mathrm{~S}_{1,2}(1-x)+\ln (x) \operatorname{Li}_{2}(1-x)\right] \\
& x^{2} \otimes \frac{\mathrm{S}_{1,2}(1-x)}{1-x}=x^{2}\left[\mathrm{~S}_{2,2}(1-x)-3 \mathrm{~S}_{1,3}(1-x)\right] \\
& +\left[-x^{2} \ln (x)+\frac{1}{2}+x-\frac{3}{2} x^{2}\right] \mathrm{S}_{1,2}(1-x) \\
& +\frac{x^{2}}{4} \ln ^{3}(x)-\frac{x}{4} \ln ^{2}(x)-\frac{x}{2} \ln (x)-\frac{1}{2} x(1-x)
\end{aligned}
$$

Acknowledgments: This work was supported in part by DFG Sonderforschungsbereich Transregio 9, Computergestützte Theoretische Physik. 


\section{References}

[1] see e.g. : A. Akhundov, D. Bardin, and T. Riemann, Nucl. Phys. B276 (1986) 1;

S. Jadach and B. Ward, Comput. Phys. Commun. 56 (1990) 351, 66 (1991) 276; 124 (2000) 233 ;

M. Consoli and M. Greco, Nucl. Phys. B186 (1981) 519.

[2] W. Beenakker, F. A. Berends and W. L. van Neerven, Print-89-0445 (Leiden) Proc. Workshop on Electroweak Radiative Corrections, Ringberg Castle, Germany, Apr 3-7, 1989, p. 3.

[3] J. Blümlein, Z. Phys. C47 (1990) 89.

[4] D. Y. Bardin, J. Blümlein, P. Christova and L. Kalinovskaya, Nucl. Phys. B 506 (1997) 295 [arXiv:hep-ph/9612435].

[5] E. Accomando et al. [ECFA/DESY LC Physics Working Group], Phys. Rept. 299 (1998) 1 [arXiv:hep-ph/9705442];

J. A. Aguilar-Saavedra et al. [ECFA/DESY LC Physics Working Group], arXiv:hep$\mathrm{ph} / 0106315$.

[6] F. A. Berends, W. L. van Neerven and G. J. Burgers, Nucl. Phys. B297 (1988) 429 [Erratumibid. B304 (1988) 921].

[7] G. Altarelli and G. Martinelli, in J. Ellis, R.D. Peccei ( Eds.): Physics At LEP, Vol. 1, p 47.

[8] J. Blümlein, Z. Phys. C65 (1995) 293 [arXiv:hep-ph/9403342].

[9] A. Arbuzov, D. Y. Bardin, J. Blümlein, L. Kalinovskaya and T. Riemann, Comput. Phys. Commun. 94 (1996) 128 [arXiv:hep-ph/9511434].

[10] M. Jezabek, Z. Phys. C56 (1992) 285.

[11] G. Montagna, O. Nicrosini and F. Piccinini, Phys. Lett. B406 (1997) 243 [arXiv:hep$\mathrm{ph} / 9611463]$.

[12] M. Skrzypek, Acta Phys. Polon. B23 (1992) 135.

[13] M. Przybycien, Acta Phys. Polon. B24 (1993) 1105 [arXiv:hep-th/9511029].

[14] A. B. Arbuzov, Phys. Lett. B470 (1999) 252 [arXiv:hep-ph/9908361].

[15] J. Blümlein and H. Kawamura, Nucl. Phys. B708 (2005) 467 [arXiv:hep-ph/0409289].

[16] J. Blümlein and H. Kawamura, Acta Phys. Polon. B33 (2002) 3719 [arXiv:hep-ph/0207259]; [arXiv:hep-ph/0309135]; Nucl. Phys. Proc. Suppl. 116 (2003) 110 [arXiv:hep-ph/0211219].

[17] J. Blümlein, S. Riemersma and A. Vogt, Eur. Phys. J. C1 (1998) 255 [arXiv:hepph/9611214]; Nucl. Phys. Proc. Suppl. 51C (1996) 30 [arXiv:hep-ph/9608470].

[18] R. Kirschner and L. N. Lipatov, Nucl. Phys. B213 (1983) 122 ;

J. Blümlein and A. Vogt, Phys. Lett. B370 (1996) 149 [arXiv:hep-ph/9510410]; Phys. Lett. B386 (1996) 350 [arXiv:hep-ph/9606254]; Acta Phys. Polon. B27 (1996) 1309 [arXiv:hep$\mathrm{ph} / 9603450]$; 
J. Bartels, B. I. Ermolaev and M. G. Ryskin, Z. Phys. C72 (1996) 627 [arXiv:hepph/9603204];

Y. Kiyo, J. Kodaira and H. Tochimura, Z. Phys. C74 (1997) 631 [arXiv:hep-ph/9701365].

[19] V. S. Fadin, E. A. Kuraev and L. N. Lipatov, Phys. Lett. B60 (1975) 50.

[20] O. V. Tarasov, A. A. Vladimirov and A. Y. Zharkov, Phys. Lett. B93 (1980) 429;

S. A. Larin and J. A. M. Vermaseren, Phys. Lett. B303 (1993) 334 [arXiv:hep-ph/9302208].

[21] W. Furmanski and R. Petronzio, Z. Phys. C11 (1982) 293.

[22] J. Blümlein and A. Vogt, Phys. Rev. D58 (1998) 014020 [arXiv:hep-ph/9712546].

[23] J. Blümlein and H. Kawamura, Phys. Lett. B553 (2003) 242 [arXiv:hep-ph/0211191].

[24] A. Arbuzov and K. Melnikov, Phys. Rev. D66 (2002) 093003 [arXiv:hep-ph/0205172].

[25] J. Blümlein, V. Ravindran and W. L. van Neerven, Nucl. Phys. B586 (2000) 349 [arXiv:hep$\mathrm{ph} / 0004172]$.

[26] D. J. Gross and F. Wilczek, Phys. Rev. D9 (1974) 980.

[27] G. Altarelli and G. Parisi, Nucl. Phys. B126 (1977) 298.

[28] A. De Rujula, R. Petronzio and A. Savoy-Navarro, Nucl. Phys. B154 (1979) 394.

[29] E. A. Kuraev and V. S. Fadin, Sov. J. Nucl. Phys. 41 (1985) 466 [Yad. Fiz. 41 (1985) 733].

[30] L. W. Mo and Y. S. Tsai, Rev. Mod. Phys. 41 (1969) 205.

[31] J. Blümlein, G. Levman and H. Spiesberger, J. Phys. G19 (1993) 1695.

[32] W. Furmanski and R. Petronzio, Phys. Lett. B97 (1980) 437;

E. G. Floratos, C. Kounnas and R. Lacaze, Nucl. Phys. B192 (1981) 417;

W. K. Tung, Nucl. Phys. B315 (1989) 378.

[33] J. Kripfganz and H. Perlt, Z. Phys. C41 (1988) 319;

H. Spiesberger, Phys. Rev. D52 (1995) 4936 [arXiv:hep-ph/9412286];

A. D. Martin, R. G. Roberts, W. J. Stirling and R. S. Thorne, Eur. Phys. J. C39 (2005) 155 [arXiv:hep-ph/0411040].

[34] J. Ohnemus, S. Rudaz, T. F. Walsh and P. M. Zerwas, Phys. Lett. B334 (1994) 203 [arXiv:hep-ph/9406235];

J. Blümlein and E. Boos, Nucl. Phys. Proc. Suppl. 37B (1994) 181.

[35] J. Blümlein, Comput. Phys. Commun. 159 (2004) 19 [arXiv:hep-ph/0311046].

[36] J. Blümlein and S. Kurth, Phys. Rev. D60 (1999) 014018 [arXiv:hep-ph/9810241]. 